\title{
Morphology of Active Deformable 3D Droplets
}

\author{
Liam J. Ruske๑* and Julia M. Yeomans \\ Rudolf Peierls Centre For Theoretical Physics, University of Oxford, Oxford OX1 3PU, United Kingdom
}

(Received 16 October 2020; revised 11 January 2021; accepted 25 February 2021; published 1 April 2021)

\begin{abstract}
We numerically investigate the morphology and disclination line dynamics of active nematic droplets in three dimensions. Although our model incorporates only the simplest possible form of achiral active stress, active nematic droplets display an unprecedented range of complex morphologies. For extensile activity, fingerlike protrusions grow at points where disclination lines intersect the droplet surface. For contractile activity, however, the activity field drives cup-shaped droplet invagination, run-and-tumble motion, or the formation of surface wrinkles. This diversity of behavior is explained in terms of an interplay between active anchoring, active flows, and the dynamics of the motile disclination lines. We discuss our findings in the light of biological processes such as morphogenesis, collective cancer invasion, and the shape control of biomembranes, suggesting that some biological systems may share the same underlying mechanisms as active nematic droplets.
\end{abstract}

DOI: 10.1103/PhysRevX.11.021001

Subject Areas: Biological Physics, Soft Matter

\section{INTRODUCTION}

Active particles use energy from their surroundings to do work. Examples range from eukaryotic cells, bacterial suspensions, and motor proteins to active colloids and shaken granular rods [1,2]. Active systems have recently received considerable attention in the community because of their potential in designing mesoscopic engines, as a way of interpreting biological mechanics, and as examples of nonequilibrium statistical physics [3,4]. Here, we focus on dense active systems and, in particular, the continuum theory of active nematics, which describes active systems with hydrodynamic interactions. The archetypal experimental example is microtubules driven by kinesin motor proteins $[5,6]$. Other active nematics include myosindriven actin-microtubule networks [7], swimming bacterial swarms, and confluent eukaryotic cells [8-11].

A key property of active nematics, which distinguishes them from passive liquid crystals, is active turbulence. This turbulence is a chaotic flow state characterized by strong vorticity and motile topological defects which are continually created and destroyed. Considerable experimental and theoretical work is devoted to understanding the properties of active turbulence, and the associated topological defects, in two dimensions. More recently, it has proved possible to design an active material that allows

\footnotetext{
*liam.ruske@physics.ox.ac.uk
}

Published by the American Physical Society under the terms of the Creative Commons Attribution 4.0 International license. Further distribution of this work must maintain attribution to the author(s) and the published article's title, journal citation, and DOI. imaging of a three-dimensional active nematic and, in particular, the associated motile disclination loops and lines [12]. This imaging is achieved by dispersing forcegenerating microtubule bundles in a passive colloidal liquid crystal based on filamentous viruses. The temporal evolution of disclination lines is measured using light-sheet microscopy, revealing that the primary topological excitations in bulk active nematics are charge-neutral disclination loops.

Numerical simulations reveal that flows and morphological dynamics of disclination lines in three-dimensional (3D) active nematics are governed by the local director profile surrounding the disclination line and that defect loops in extensile systems are generally formed via the well-known bend instability [13]. Three-dimensional active nematic turbulence and disclination line dynamics in spherical confinement is also investigated using numerical modeling in both achiral [14] and chiral systems [15,16]. Enforcing strong in-plane surface alignment allows the formation of defects on the surface and is used to highlight the coupling of surface and bulk topological defect dynamics.

The theories of active materials are increasingly being used to describe biological systems in two dimensions, with examples including biofilm initiation [17], topological defects in cell monolayers [8-10], and epithelial expansion [18]. This use suggests that in three dimensions there may be relevance to the collective motion of groups of cells, in morphogenesis, or to the growth and spread of tumors. Therefore, to underpin extension of these approaches to $3 \mathrm{D}$, in this paper, we investigate active, self-deforming droplets in three dimensions, describing the interplay between disclination dynamics and droplet morphology. We find that extensile droplets form protrusions at points 
where disclination lines reach the surface. In contractile droplets, however, disclinations lead to a wrinkled drop surface. Moreover, for small, contractile droplets, we find that invagination, to form a cuplike configuration, can be driven by nematic activity alone. We present evidence explaining the reasons behind the different behaviors, stressing, in particular, the role of active anchoring, an effective surface alignment resulting from active flows.

We start by presenting the mathematical description of an active nematic and the equations of motion which we solve numerically and follow this presentation by a summary of 3D active nematics and the classification of disclination lines. The main results are presented in Sec. IV, which is split into four subsections: First, we investigate the nematic structure on the surface of an active droplet (Sec. IVA) and how this structure affects the dynamics of disclination lines in the bulk of spherical droplets (Sec. IV B). We then move on to show how active forces can deform the interface by highlighting mechanisms causing droplet deformations triggered by both extensile (Sec. IV C) and contractile (Sec. IV D) activity. The last section of the paper summarizes the key results and points out possible connections to biological systems.

\section{EQUATIONS OF MOTION}

We summarize the continuum equations of motion describing the dynamics of a deformable, nematic droplet in an isotropic fluid background. In the absence of activity, the system is described by a free energy $\mathcal{F}=\int f d \mathbf{V}=$ $\int f_{\mathrm{GL}}+f_{\mathrm{LC}} d \mathbf{V}$. The first contribution, which controls the formation of the nematic droplet, is chosen to take the Ginzburg-Landau form

$$
f_{\mathrm{GL}}=\frac{\kappa^{*}}{2}\left(-\varphi+\varphi^{3}-\epsilon^{2} \nabla^{2} \varphi\right)^{2}+\frac{K_{\varphi}}{2}(\nabla \varphi)^{2} .
$$

This describes phase separation into two stable phases with concentrations $\varphi= \pm 1$ with an interface of width $\epsilon$ separating the two phases $[19,20]$. The bending rigidity $\kappa$ of the interface is related to $\kappa^{*}$ by $\kappa=\left(2 \epsilon^{3} / 3 \sqrt{2}\right) \kappa^{*}$. The final term penalizes gradients in the concentration field and introduces a surface tension $\sigma$. We outline the mechanical properties of the interface generated with this phase-field modeling approach in Appendix A.

The second contribution to the free energy density is

$$
\begin{aligned}
f_{\mathrm{LC}}= & A_{\mathrm{LC}}\left\{\frac{1}{2}\left(1-\frac{\eta(\varphi)}{3}\right) \operatorname{tr}\left(\mathbf{Q}^{2}\right)-\frac{\eta(\varphi)}{3} \operatorname{tr}\left(\mathbf{Q}^{3}\right)\right. \\
& \left.+\frac{\eta(\varphi)}{4} \operatorname{tr}\left(\mathbf{Q}^{2}\right)^{2}\right\}+\frac{1}{2} K_{\mathrm{LC}}(\nabla \mathbf{Q})^{2},
\end{aligned}
$$

which includes the usual Landau-de Gennes bulk energy of the liquid crystal expanded in terms of the nematic tensor $\mathbf{Q}$ which has elements $Q_{i j}=3 S_{0} / 2\left(n_{i} n_{j}-\delta_{i j} / 3\right)$, where $S_{0}$ is the magnitude and $\mathbf{n}$ the direction of the nematic order, and a term which penalizes elastic deformations of the director field [21]. $A_{\mathrm{LC}}$ sets the bulk energy scale, and we use the one-elastic-constant approximation which assigns the same elastic constant $K_{\mathrm{LC}}$ to twist, splay, and bend deformations. The function $\eta(\varphi)$, which quantifies the dependence of bulk energy on the local liquid crystal concentration, predicts a first-order phase transition between a nematic and an isotropic phase of the liquid crystal at the value $\eta_{c}=2.7$. We define $\eta(\varphi):=\eta_{c}+\eta_{1} \varphi$ and choose a value of $\eta_{1}$ that ensures that the system forms a nematic droplet $(\varphi=+1)$ which is surrounded by isotropic fluid $(\varphi=-1)$. Alternative expressions for $\eta(\varphi)$ or the liquid crystal bulk energy expressions are used in other work [22,23], resulting in qualitatively the same dynamics, as long as the droplet in the nematic phase $\left[\eta(\varphi=1)>\eta_{c}\right]$ and the environment is isotropic $\left[\eta(\varphi=-1)<\eta_{c}\right]$.

Since the total concentration $\int \varphi d V$ is assumed to stay constant, diffusive transport follows model-B dynamics, and the time evolution of $\varphi$ is governed by the following reaction-diffusion equation [24]:

$$
\left(\partial_{t}+\mathbf{u} \cdot \nabla\right) \varphi=\Gamma_{\varphi} \nabla^{2} \mu .
$$

Here, $\mathbf{u}$ is the velocity field, and the mobility $\Gamma_{\varphi}$ quantifies how fast $\varphi$ responds to gradients in the chemical potential $\mu=\delta \mathcal{F} / \delta \varphi$.

Unlike the total concentration, the local nematic alignment $\mathbf{Q}$ is not a conserved quantity, and its time evolution follows modified model-A dynamics [25]:

$$
\left(\partial_{t}+\mathbf{u} \cdot \nabla\right) \mathbf{Q}-\mathbf{S}=\Gamma_{Q} \mathbf{H},
$$

where $\Gamma_{Q}$ is the rotational diffusivity and $\mathbf{H}$, the molecular field, is given by

$$
\mathbf{H}=-\left[\frac{\delta \mathcal{F}}{\delta \mathbf{Q}}-\frac{1}{3} \mathbf{I} \operatorname{tr}\left(\frac{\delta \mathcal{F}}{\delta \mathbf{Q}}\right)\right] .
$$

Rodlike particles can not only be advected by the fluid, but also rotate in response to flow gradients. This behavior is accounted for by the corotational term [25]

$$
\begin{aligned}
S_{i j}= & \left(\xi D_{i k}+\Omega_{i k}\right)\left(Q_{k j}+\frac{\delta_{k j}}{3}\right)+\left(Q_{i k}+\frac{\delta_{i k}}{3}\right)\left(\xi D_{k j}-\Omega_{k j}\right) \\
& -2 \xi\left(Q_{i j}+\frac{\delta_{i j}}{3}\right) Q_{k l} W_{l k}
\end{aligned}
$$

where $D_{i j}=\left(\partial_{j} u_{i}+\partial_{i} u_{j}\right) / 2$ and $\Omega_{i j}=\left(\partial_{j} u_{i}-\partial_{i} u_{j}\right) / 2$ are the symmetric and antisymmetric parts of the velocity gradient tensor $W_{i j}=\partial_{i} u_{j}$, respectively. The parameter $\xi$ determines whether the director aligns with, or tumbles in, a shear flow. Its value depends on the molecular details of the liquid crystal, and it is related to the flow alignment parameter $\lambda$ defined within Ericksen-Leslie theory by 
$\lambda=\left(3 S_{0}+4 / 9 S_{0}\right) \xi$. This work mostly focuses on flow tumbling nematics, for which the flow alignment parameter $\lambda<1$.

We assume that the buildup of active flows occurs over timescales which are much longer than any viscoelastic relaxation time of the system. We therefore consider the liquid limit and solve the incompressible Navier-Stokes equations to obtain the flow field $\mathbf{u}$ :

$$
\begin{gathered}
\nabla \cdot \mathbf{u}=0, \\
\rho\left(\partial_{t}+\mathbf{u} \cdot \nabla\right) \mathbf{u}=\nabla \cdot \Pi,
\end{gathered}
$$

where the stress tensor $\Pi=\Pi_{\text {viscous }}+\Pi_{\text {elastic }}+\Pi_{\text {capillary }}+$ $\Pi_{\text {active }}$. The passive contributions, well known from liquid crystal hydrodynamics [26], are

$$
\begin{gathered}
\Pi_{\text {viscous }}=2 \eta \mathbf{D}, \\
\Pi_{\text {capillary }}=(f-\mu \varphi) \mathbf{I}-\nabla \varphi\left(\frac{\partial f}{\partial(\nabla \varphi)}\right)+\nabla \varphi \nabla\left(\frac{\partial f}{\partial\left(\nabla^{2} \varphi\right)}\right) \\
-\nabla \nabla \varphi\left(\frac{\partial f}{\partial\left(\nabla^{2} \varphi\right)}\right), \\
\Pi_{\text {elastic }}=-p \mathbf{I}-\xi[\mathbf{H} \tilde{\mathbf{Q}}+\tilde{\mathbf{Q}} \mathbf{H}-2 \tilde{\mathbf{Q}} \operatorname{tr}(\mathbf{Q H})] \\
+\mathbf{Q H}-\mathbf{H Q}-\nabla \mathbf{Q}\left(\frac{\partial f}{\partial(\nabla \mathbf{Q})}\right),
\end{gathered}
$$

where $\rho$ is the density, $\eta$ the viscosity, $p$ the bulk pressure, and $\tilde{\mathbf{Q}}=\left(\mathbf{Q}+\frac{1}{3} \mathbf{I}\right)$. In addition to the passive terms, the stress due to the dipolar flow fields produced by the active particles is [27]

$$
\Pi_{\text {active }}=-\zeta \mathbf{Q},
$$

where $\zeta$ quantifies the magnitude of active stress. For extensile activity, $\zeta>0$, nematic particles direct the fluid outward along their elongated direction and inward along the two perpendicular axes. The flow direction is reversed for contractile activity, $\zeta<0$.

We solve the equations of motion using a hybrid latticeBoltzmann-finite-difference method. This method involves solving Eqs. (3) and (4) using finite-difference methods and Eqs. (7) and (8) using a lattice-Boltzmann algorithm $[21,22,25,28]$. Simulations are performed on a threedimensional lattice of size $100 \times 100 \times 100$, and discrete space and time steps are chosen as unity. We use periodic boundary conditions for the simulation box, and the system initially consists of a spherical, nematic drop of radius $R=30(\varphi=+1)$ embedded in an isotropic fluid $(\varphi=-1)$, unless otherwise stated. Initially, we let the system relax for $t=500$ time steps such that the droplet interface and the nematic tensor $\mathbf{Q}$ reach their equilibrium profile before we switch on activity. We use the following parameter set for all simulations: $\rho=1, p=0.25, \Gamma_{Q}=0.1, \Gamma_{\varphi}=0.2$, $\kappa^{*}=0.1, \epsilon=1.4, A_{\varphi}=0.1, \eta_{1}=0.3, \xi=0.1$, and $\eta=$ $1 / 3$ in lattice-Boltzmann units. For spherical droplets in Secs. IV A and IV B, we use $K_{\mathrm{LC}}=0.1, K_{\varphi}=0.4, A_{\mathrm{LC}}=$ 0.75 , and $|\zeta|=0.01$. For soft droplets in Secs. IV C and IVD, we use the following parameters, unless otherwise stated: $K_{\mathrm{LC}}=0.2, K_{\varphi}=0.2, A_{\mathrm{LC}}=1.5$, and $|\zeta|=0.02$. In Appendix B, we provide a mapping of the simulation parameters to physical units.

\section{DISCLINATION LINES IN THREE-DIMENSIONAL ACTIVE NEMATICS}

Active stress leads to an instability of the nematic phase (Fig. 1). This hydrodynamic instability constantly pushes the system out of equilibrium, leading to a chaotic steady state termed active turbulence [29]. 3D active turbulence is characterized by spatiotemporally chaotic flows and the presence of disclination lines which constantly undergo transformation events such as breakup, recombination, nucleation, and annihilation [14]. In bulk systems, disclination lines typically form closed, charge-neutral loops [12]. However, in the presence of a confining interface, as for nematic droplets, disclination lines can also terminate at the boundary, and the dynamics of the resultant defects on the surface is coupled to disclination line dynamics in the bulk by elastic interactions and flows.

Unlike in two-dimensional active nematics, where $\pm 1 / 2$ defects carry topological charge and can therefore nucleate or annihilate only in pairs of opposite charge, disclination lines in three dimensions can continuously transform from a local $-1 / 2$ configuration (in the plane perpendicular to the line) into $\mathrm{a}+1 / 2$ configuration through an intermediate twist winding as indicated in Fig. 2. As one moves around the core of a disclination in the plane perpendicular to the local disclination line segment, the director field winds around a specific axis, the rotation vector $\boldsymbol{\Omega}$ (black arrows) by an angle $\pi$. The angle $\beta$ between $\boldsymbol{\Omega}$ and the local line tangent $\mathbf{t}$ (yellow arrow) is called the twist angle and can be used to locally characterize the disclination line. For $-1 / 2$ $(+1 / 2)$ wedge-type defects, the twist angle corresponds to $\beta=0(\pi)$, while line segments with local twist-type defects are indicated by $\beta=\pi / 2$. (a)

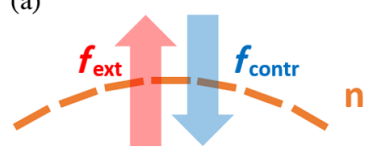

(b)

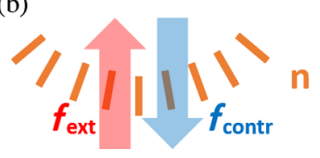

FIG. 1. Bend (a) and splay (b) perturbations of the director field n cause active forces which set up active flows which further enhance or stabilize the respective perturbation. Forces in extensile or contractile systems are denoted by red or blue arrows, respectively. It is apparent that extensile (contractile) systems are unstable to bend (splay) deformations. 


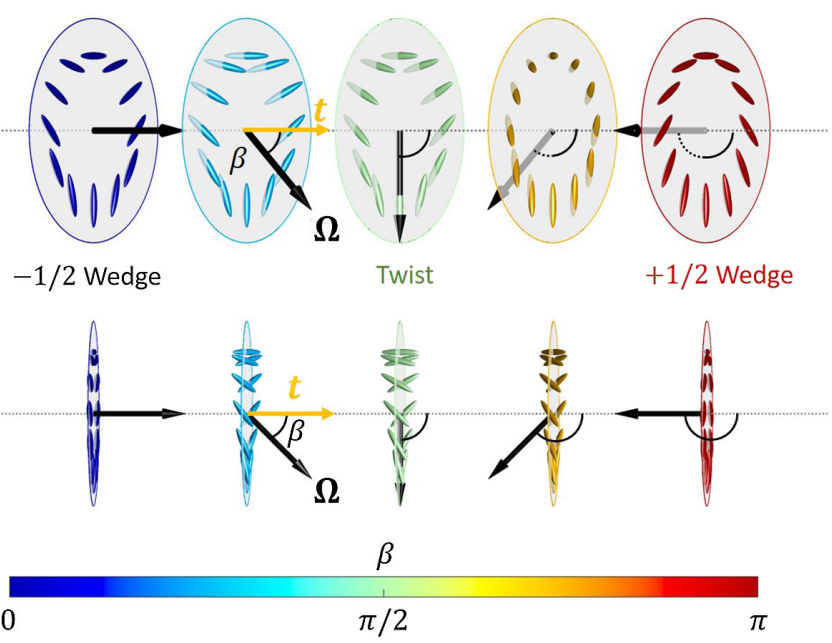

FIG. 2. A local $-1 / 2$ wedge can continuously transform into a $+1 / 2$ wedge via an intermediate twist disclination [12]. The director field winds about the rotation vector $\boldsymbol{\Omega}$ (black arrows) by an angle $\pi$. The angle between the rotation vector $\boldsymbol{\Omega}$ and the local disclination line tangent $\mathbf{t}$ (yellow arrow), called the twist angle $\beta$, varies continuously along a disclination line. Twist disclinations correspond to $\beta=\pi / 2$ and $+1 / 2$ and $-1 / 2$ wedge disclinations to $\beta=\pi$ and 0 , respectively.

Because of activity, disclination lines act as selfpropelled entities moving through the fluid. Based on a simplified model neglecting elastic interactions, each disclination line segment can be associated with a local selfpropulsion velocity which depends on the local director profile [13]. The self-propulsion velocity component perpendicular to the local tangent of the line depends on twist angle $\beta$ as

$$
v_{\perp}^{\mathrm{SP}} \propto(1-\cos \beta)^{2} .
$$

Thus, unlike in two-dimensional active turbulence where the dynamics is dominated by two species of quasiparticles ( $\pm 1 / 2$ defects), disclination lines in three-dimensional active turbulence act as quasiparticles with an intrinsic degree of freedom, the twist angle $0 \leq \beta \leq \pi$. Line segments with $\beta=0$ are passive, while $\beta=\pi$ line segments are most active in pushing around the surrounding fluid. Please refer to Appendix $\mathrm{C}$ for further information about the detection of disclination lines and the calculation of twist angle $\beta$ on a finite grid used for the lattice-Boltzmann simulation.

\section{RESULTS}

\section{A. Activity leads to preferred director alignment at an interface}

In 2D active nematics, the flow induced by active stresses leads to an alignment of the director at an interface with an isotropic phase [23]. This active anchoring of the director field is parallel to the interface for extensile stress and perpendicular for contractile stress. For $2 \mathrm{D}$ systems, the active alignment can be significant, but it is not immediately obvious whether the effect persists in a 3D geometry or how it is affected by any defects present on the surface.

Therefore, to investigate the effects of active anchoring in $3 \mathrm{D}$, we measure the angle $\theta$ between the director field $\mathbf{n}$ and the surface normal at the interface of spherical droplets. Since there are more possible configurations for in-plane alignment $(\theta=\pi / 2)$ than for perpendicular alignment $(\theta=0)$, we use the distribution of $\cos \theta$ to quantify alignment effects. A randomly aligned director field results in a uniformly distributed $\cos \theta \sim \mathcal{U}[0,1]$, while preferred perpendicular or in-plane alignment leads to bias of the distribution toward 1 or 0 , respectively. The results in Fig. 3 show clear evidence that both extensile and contractile

(a)
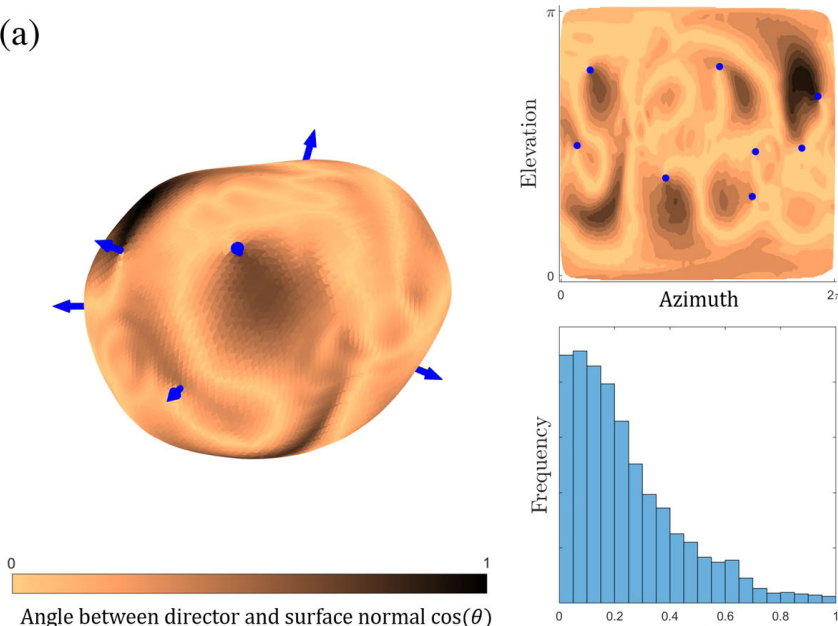

Angle between director and surface normal $\cos (\theta)$

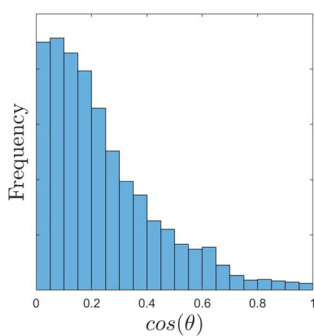

(b)

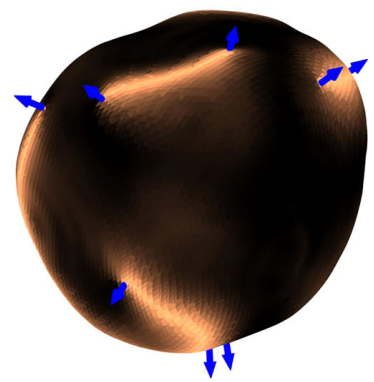

Angle between director and surface normal $\cos (\theta)$
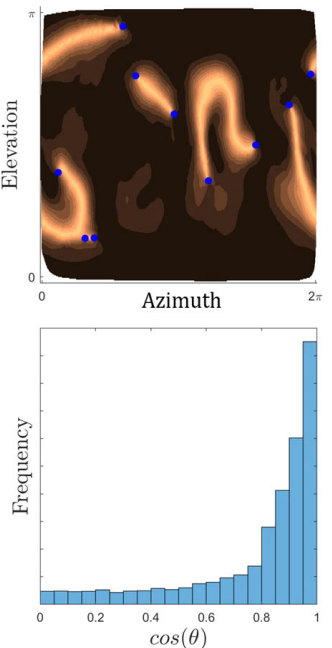

FIG. 3. Surface alignment of the director field for extensile (a) and contractile activity (b). Surface defects, shown as blue arrows, are connected via disclination lines running through the bulk. The surface alignment is indicated by the color bar, where orange (black) indicates in-plane (perpendicular) director alignment with respect to the interface. Upper right: surface alignment in projection. Lower right: distribution of surface angle $\cos (\theta)$ over the total surface area. 


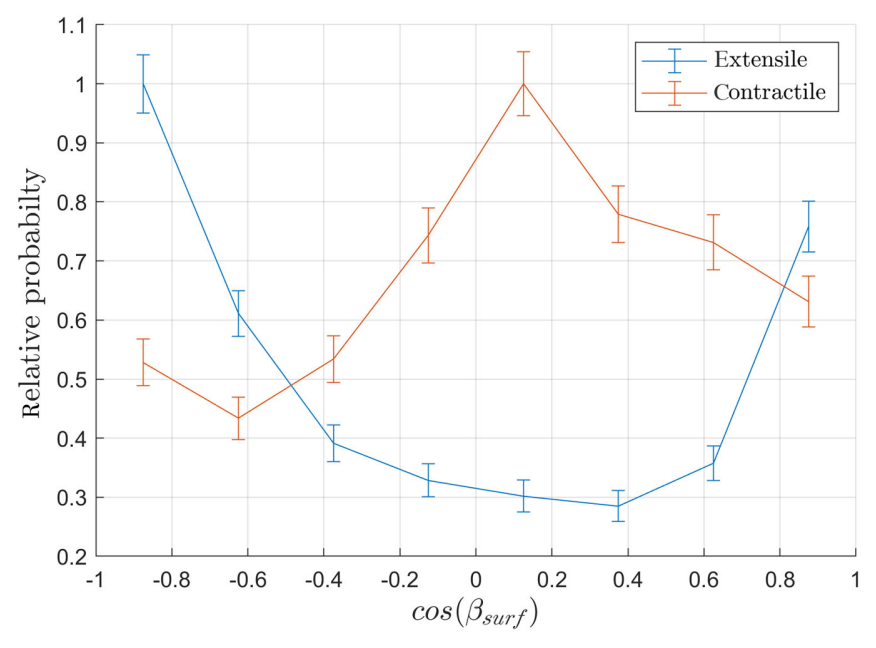

FIG. 4. Distribution of twist angles of surface defects $\beta_{\text {surf }}$, i.e., at positions where disclination lines intersect the droplet interface, obtained from a time average in the active turbulent regime. Surface defects are preferentially wedgelike (twistlike) in extensile (contractile) systems where active anchoring favors inplane (normal) director alignment. The relative probability is given by $N_{i} / \max \left(\left\{N_{i}\right\}\right)$, where $N_{i}$ is the number of observations in bins of range $X_{i} \pm h / 2$, and error bars denote the bin error $\sqrt{N_{i}} / \max \left(\left\{N_{i}\right\}\right)$. The averaging is performed over $T=250000$ time steps, which is much longer than the average time it takes for a disclination line to move across the droplet $\tau_{\mathrm{SP}} \approx 15000$.

activity lead to strong active anchoring on the surface, which weakly depends on the flow-alignment parameter $\lambda$ (Fig. S1 [30]).

The director orientation also shows signatures of the places where disclination lines end at the surface. Consider first the case of extensile activity. The director lies in plane over most of the surface, although there are localized regions with perpendicular alignment [see Fig. 3(a) and Movie S1 [30]]. The disclination lines present in the bulk of the droplet create quasi-two-dimensional defects at the positions where they terminate at the interface which we shall term surface defects. The distribution of the twist angle $\beta$ of surface defects has distinct peaks at $\beta=0$ and $\beta=\pi$, showing that most defects on the surface are of wedge type (Fig. 4), corresponding approximately to $2 \mathrm{D}+$ $1 / 2$ and $-1 / 2$ configurations. There are fewer twist-type surface defects with $\beta \approx \pi / 2$, as these introduce a perpendicularly aligned region in the vicinity of the defect, which is suppressed by active anchoring.

A two-dimensional nematic sheet confined to the surface of a sphere always has at least four $+1 / 2$ defects present, as the total topological charge is conserved and must add up to two, the Euler-characteristic $\chi$ of a sphere. Active anchoring does not confine the director field to the surface everywhere, so the topological charge is not strictly conserved.

By contrast, contractile activity leads to a remarkable stripe pattern in the director alignment on the surface.

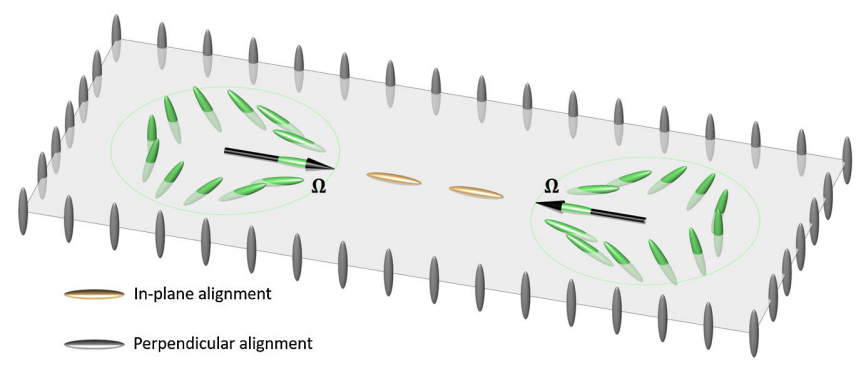

FIG. 5. Adjacent twist-type surface defects (green) tend to align antiparallel to each other in contractile systems to minimize the region of in-plane alignment. This alignment results in lines of planar surface anchoring between the defects (orange directors).

While most parts of the surface show strong perpendicular (homeotropic) active alignment, this alignment is interspersed with thin stripes of clear in-plane ordering [see Fig. 3(b) and Movie S1 [30]]. The stripes start and terminate at surface defects which are mostly twist type with $\beta \approx \pi / 2$ (Fig. 4). Wedge-type surface disclinations with $\beta=0, \pi$ are suppressed, as they would create a region of in-plane alignment in the vicinity of the defect. Twisttype disclinations, on the other hand, introduce only a small region of in-plane alignment along one specific direction (Fig. 2) and are, therefore, favored. Adjacent twist-type disclinations tend to align with their rotation vectors antiparallel, in a way that minimizes the area of unfavorable in-plane alignment, thereby creating the observed stripe pattern (Fig. 5).

Perfect perpendicular alignment at the surface prevents disclination lines in the bulk terminating there, as all disclination lines introduce some degree of in-plane alignment at the surface. Indeed, because topological charge is conserved, a sphere with perfect homeotropic alignment at the interface forces the system to form $a+1$ hedgehog defect in the bulk. Like in two dimensions where $a+1$ disclination splits into two $+1 / 2$ defects, in three dimensions the hedgehog defect forms a small +1 defect loop in the bulk $[13,14]$. However, +1 defect loops are associated with large elastic energy of the liquid crystal which usually cannot be overcome by active anchoring.

\section{B. Disclination line dynamics in spherical droplets}

We now focus on disclination line dynamics inside spherical droplets which are essentially undeformed by active forces. In the regime of active turbulence, disclination lines constantly form and annihilate. Previous investigations of active turbulence in bulk systems find that the dominant excitations of three-dimensional active nematics are charge-neutral disclination loops which undergo complex dynamics and recombination events [12]. In droplets, however, disclination lines do not need to form loops, as they can also exist as growing or shrinking half-circles or lines which terminate at the surface at positions $\left[\mathbf{x}_{1}, \mathbf{x}_{2}\right]$. The distance between end points (surface defects) of 
(a)

(b)
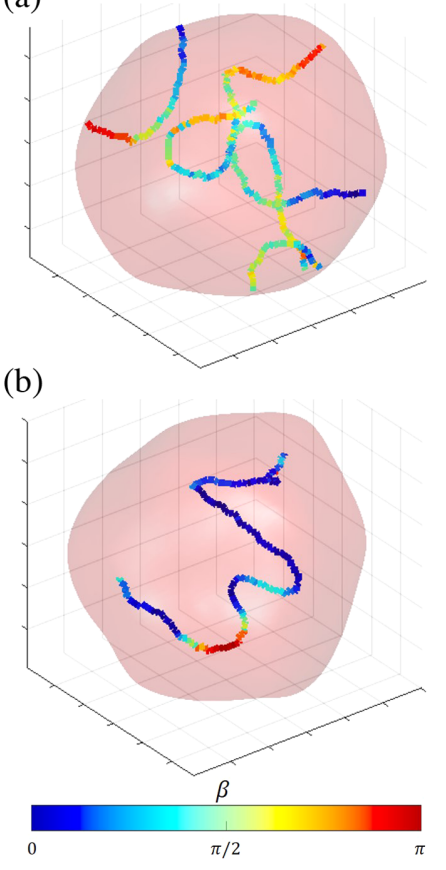

(c)

(d)
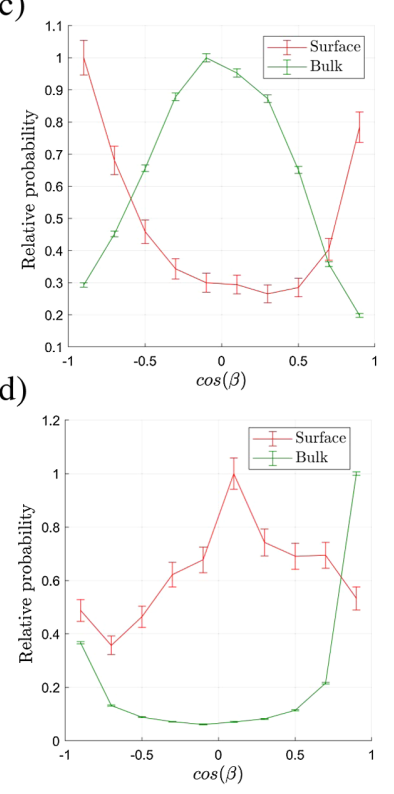

FIG. 6. Snapshots of disclination lines in an (a) extensile and (b) contractile droplet. The color bar shows the twist angle $\beta$ which characterizes the director configuration which varies continuously along the line. (c) As a consequence of active anchoring, for extensile systems, disclinations close to the surface tend to be wedge type, whereas those in the bulk tend to be twist disclinations as is evident from the distribution of $\beta$. (d) For contractile systems, disclinations close to the surface slightly tend to be twist type, whereas those in the bulk tend to be wedge disclinations. Averaging in (c) and (d) performed over $T=$ 250000 time steps. The noisiness of the colors seen for some disclination line segments in (a) occurs because of a finite grid resolution and does not significantly impact the statistics (see Appendix C).

disclination lines $D=\left|\mathbf{x}_{1}-\mathbf{x}_{2}\right|$ first scales linearly with the total length $L$ of lines before plateauing at a finite value as the lines increase in length. For disclination lines which are short compared to the droplet radius $(L<R)$, the mean end point separation scales as $D \approx(2 / \pi) L$, showing that the lines mainly nucleate or annihilate at the droplet's surface as half-circles. The end points of very long disclination lines $(L \gg R)$ are randomly distributed over the droplet's surface, so the mean separation of end points converges toward $D / R \approx 4 / 3$ (Fig. S2 [30]). The local properties of disclination lines can be classified by the twist angle $\beta$, which varies continuously along the line. We find that the director profiles of disclination lines close to the surface are heavily influenced by active anchoring which favors wedge- (twist)-type disclination lines for extensile (contractile) activity (Fig. 6 and Movie S1 [30]). This result is in contrast to the bulk of the drop, where extensile (contractile) activity favors twist- (wedge)-type disclinations. The distributions of $\beta$ at the surface and in the bulk

(a)

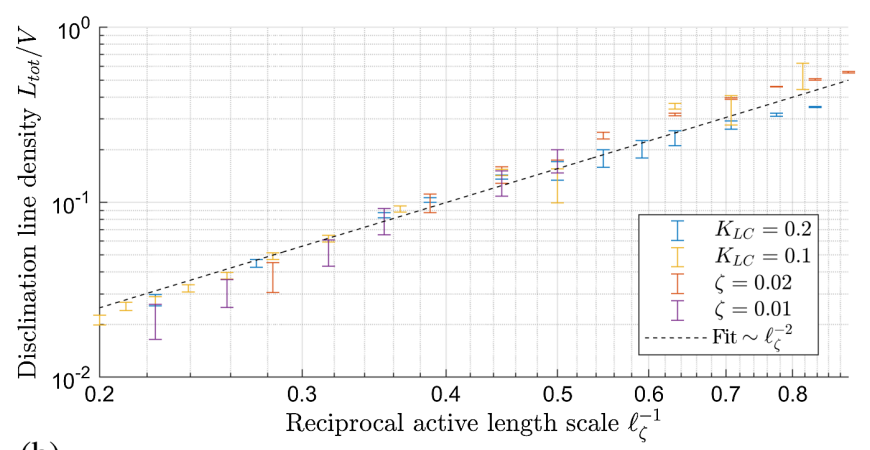

(b)

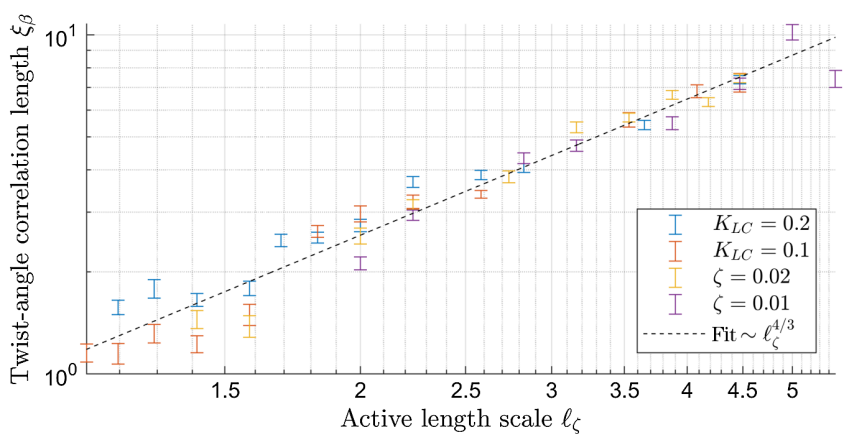

(c)

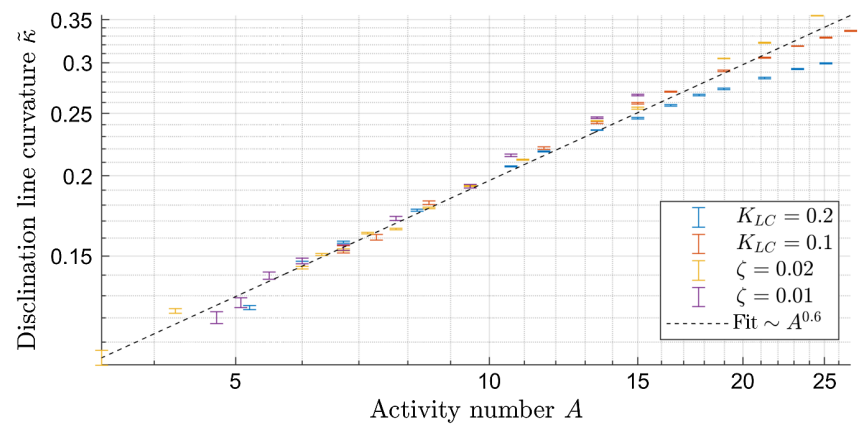

FIG. 7. (a) Mean area density of disclination lines, defined as the total length $L_{\text {tot }}$ of all disclination lines divided by the volume $V$, as a function of the inverse active length scale $1 / \ell_{\zeta}=\sqrt{\zeta / K_{\mathrm{LC}}}$. Either $K_{\mathrm{LC}}$ or $\zeta$ are varied, while the other parameter is set to a fixed value, which is indicated by the legend entries. (b) Persistence length $\xi_{\beta}$ of the twist angle $\beta$ as a function of the active length scale. (c) Mean curvature $\tilde{\kappa}$ of disclination lines as a function of activity number $A=R / \ell_{\zeta}$. Averaging performed over $T=20000$ time steps.

are compared for extensile and contractile droplets in Figs. 6(c) and 6(d), respectively. The excess of twist disclinations in extensile bulk systems has been recently confirmed by both experiments and simulations $[12,13]$.

Behind the apparent disorder of three-dimensional active turbulence, we identify an active length scale $\ell_{\zeta} \propto$ $\sqrt{K_{\mathrm{LC}} / \zeta}$ which controls the average disclination line density and coincides with the active length scale governing the decay of the vorticity correlations in twodimensional active turbulence [3]. Figure 7 shows the area 
density of disclination lines, defined as the total length $L_{\text {tot }}$ of all disclination lines divided by the droplet volume $V$, as a function of $K_{\mathrm{LC}}$ and $\zeta$, confirming that it scales with the active length scale as $\ell_{\zeta}^{-2}$. Moreover, measuring the mean variation of twist angle $\beta$ along disclination lines shows an exponential convergence toward a plateau with a characteristic length scale $\xi_{\beta}$ (Fig. S3 [30]). We find that, in the turbulent regime, the correlation length $\xi_{\beta}$ of twist angle $\beta$ scales with the active length scale as $\xi_{\beta} \propto \ell_{\zeta}^{4 / 3}$. The exponent $4 / 3>1$ reflects that disclination lines are curved structures in three-dimensional space; hence, correlations do not decay linearly along lines. Based on these observations, we define the dimensionless activity number $A=R \sqrt{\zeta / K_{\mathrm{LC}}}$ as the ratio of droplet size $R$ to active length scale $\ell_{\zeta}$. The mean curvature $\tilde{\kappa}$ of disclination lines increases with activity number roughly as $\tilde{\kappa} \sim A^{0.6}$. Deviations at large activity $A$, equivalent to small active length scale $\ell_{\zeta}$, are caused by the finite grid size of the simulation as $\ell_{\zeta}$ approaches the grid resolution limit $\Delta x$.

Because of activity, disclination lines move through the fluid with an associated transverse self-propulsion velocity $v_{\perp}^{\mathrm{SP}}$ [Eq. (13)]. In addition, they are passively advected by the surrounding flow, and, thus, the mean transverse velocity of disclination line segments is expected to follow $\left\langle v_{\perp}\right\rangle \approx v_{\text {rms }}+v_{\perp}^{\mathrm{SP}}$, where $v_{\mathrm{rms}}$ denotes the rootmean-square velocity of the fluid inside the droplet. Surprisingly, this simplified model of uncoupled, selfpropelled line segments closely matches the velocity profile we observe in turbulent droplets for large $\beta$ [Fig. 8(a)]. As disclination lines consist of line segments with varying selfpropulsion velocities, more active line segments are elastically coupled with more passive segments. Passive line segments are, thus, pulled around by elastic interactions in addition to passive advection by the flow, which is the reason for the deviation of $\left\langle v_{\perp}\right\rangle$ from the theoretical prediction for small $\beta$.

We argue that, as a consequence of active anchoring, the distribution of $\beta$ varies between disclination lines near the surface and those deep into the bulk [Fig. 8(b), solid lines]. This variation is mirrored in the average self-propulsion velocity [Fig. 8(b), diamonds]. In extensile systems, the wedge-type defects close to the surface on average move faster than the twist-type line segments in the bulk. Similarly, contractile activity causes line segments in the bulk, which are mostly wedge type, to move faster than surface defects.

\section{Extensile activity triggers the formation of fingerlike protrusions}

We now consider lower values of the surface tension such that the active flows are strong enough to deform the droplet, which leads to the formation of defect-mediated, fingerlike protrusions for extensile activity. Unlike in stiff droplets, the active flow field around disclination lines
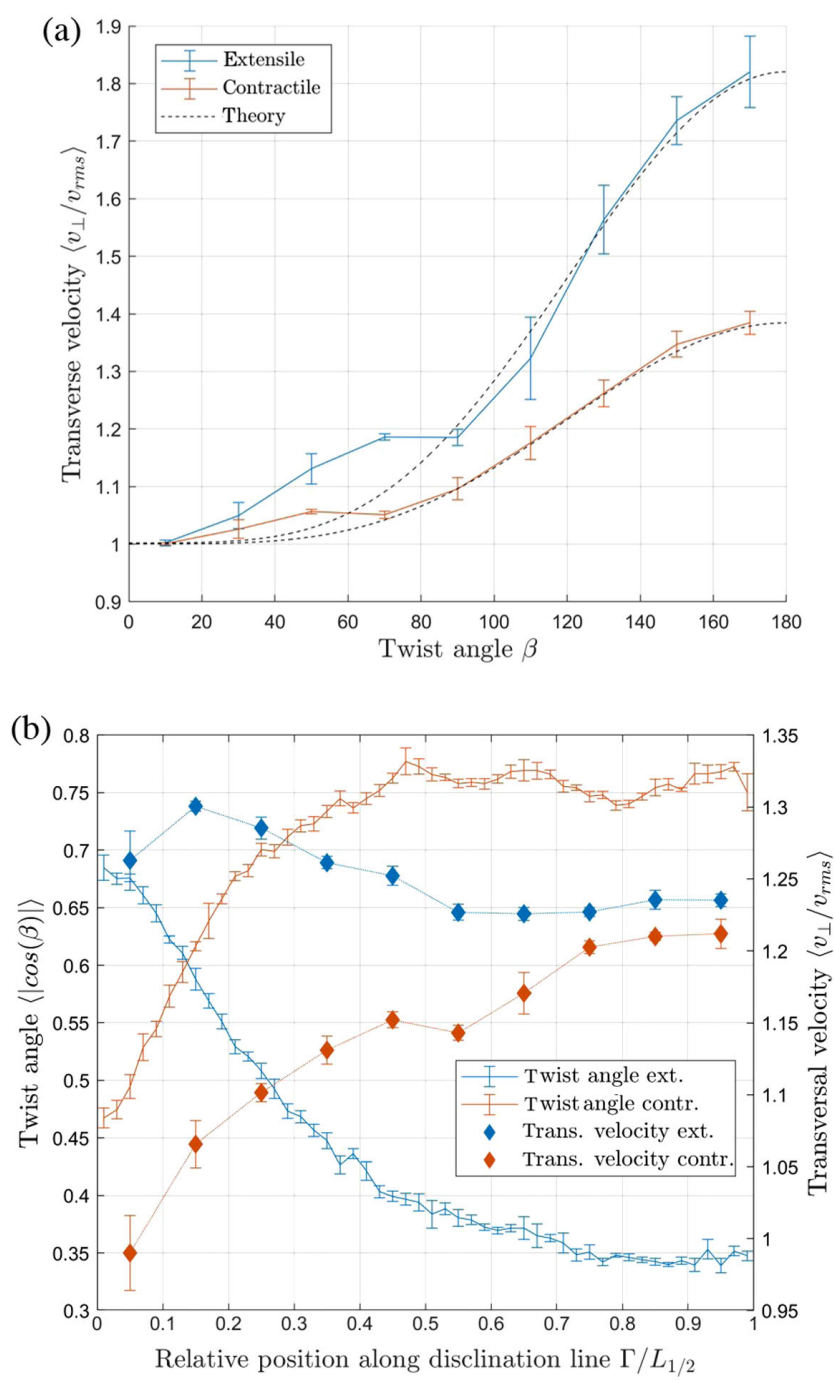

FIG. 8. (a) Mean transverse velocity $\left\langle v_{\perp}\right\rangle$ of disclination line segments as a function of local twist angle $\beta$. The transverse velocity is normalized by the average root-mean-square velocity $v_{\text {rms }}$ of the fluid. The fit is to the theoretical prediction [13], Eq. (13), with the constant of proportionality obtained from a least-square fit. (b) Mean twist angle $\langle|\cos (\beta)|\rangle$ and mean transverse velocity $\left\langle v_{\perp}\right\rangle$ as a function of the relative position along disclination lines, where values 0 and 1 denote positions close to the surface and in the bulk, respectively. The relative position is measured as the ratio of $\Gamma$, which is the path length between each point and the nearest end point of the disclination line, and $L_{1 / 2}$, which is half the total line length. Averaging performed over $T=250000$ time steps.

close to the surface can push the interface outward, creating a bulge along the self-propulsion direction of a disclination line [Fig. 9(a)]. As the disclination line continues to move outward toward the interface, the local bulge extends and forms a thin protrusion. Because of the reduced separation of interfaces compared to the spherical droplet, the properties of disclination lines inside protrusions are dominated by active anchoring which favors in-plane surface alignment. This domination causes disclination lines to span the 


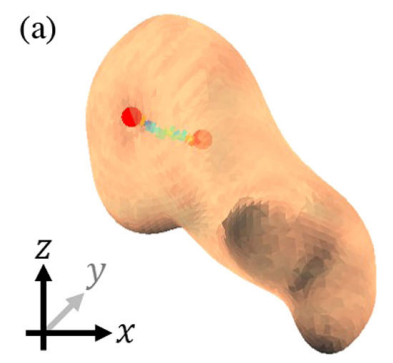

Angle between the director and the surface normal $\cos (\theta)$
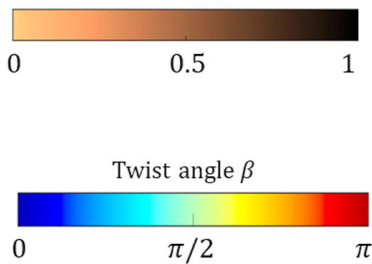

(b)

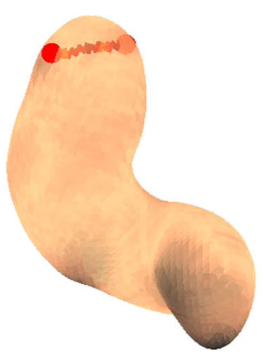

(c)

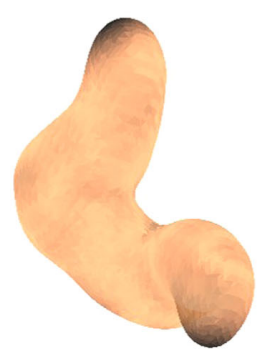

(e)

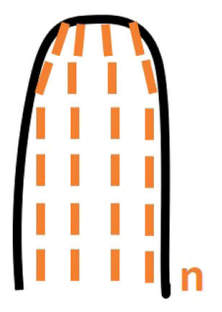

FIG. 9. Formation of fingerlike protrusions by motile disclination lines shown by three snapshots at times $t_{1}<t_{2}<t_{3}$. (a)(c) show the three-dimensional droplet shape with color coding showing the local surface alignment of the director field. Disclination line segments are colored according to the twist angle $\beta$ with the same color coding as in Fig. 6. (a) Disclination line with varying twist angle $\beta$ moves toward the interface. Active flow pushes the interface outward, creating a bulge along the selfpropulsion direction of the disclination line. (b) The small protrusion width combined with in-plane alignment at the surface stabilizes the disclination line into an almost straight configuration with $\beta \approx \pi$. (c) The disclination line moves out of the droplet, leaving behind a defect-free protrusion with an aligned director field. Disclination lines exit protrusions as nearly straight lines and violate in-plane alignment within a small area, as active anchoring is not strong enough to force the creation of a surface point defect. (d) and (e) show a schematic diagram of the director field and active forces. The red arrow in (d) denotes the self-propulsion direction of the $\beta \approx \pi$ disclination lines. As shown in (c), disclinations leave behind an area of perpendicular surface alignment (dark regions) at the end of protrusions which slowly retract due to surface tension and bending rigidity. Snapshots created from simulation with droplet size $R=15$ at times $t_{1}=71500, t_{2}=72500$, and $t_{3}=73500 ;$ see also Movie S2 [30].

width of the protrusion as a straight line with most line segments resembling wedge-type $+1 / 2$ defect profiles $(\beta \approx \pi)$, thereby enhancing the directed self-propulsion

of the disclination line even further. The $+1 / 2$ defect profile aligns along the protrusion axis, as this configuration satisfies the in-plane anchoring condition everywhere at the surface [Figs. 9(b) and 9(d)]. This quasi-two-dimensional movement of $+1 / 2$ defect lines is also observed in active nematic films confined to a channel below a critical wall separation [31]. Wedge-type $-1 / 2$ defect profiles $(\beta \approx 0)$ are not observed in protrusions, as they are passive and lack the self-propulsion necessary to form a bulge in the first place.

The $+1 / 2$ defect line eventually reaches the tip of the growing protrusion and moves out of the nematic droplet, leaving behind a homogeneous director field which is aligned along the protrusion axis [Figs. 9(c) and 9(e)]. Thereby, an area of perpendicular surface alignment is introduced at the end of protrusions, which are points of large negative mean curvature. The surface alignment $\cos (\theta)$ is, therefore, correlated to the local mean curvature of the interface (Fig. S4 [30]). In the absence of disclination lines mediating active forces, the aligned protrusions slowly retract due to surface tension and bending energy. The constant formation and retraction of droplet protrusions is also shown in Movie S2 [30].

To further quantify the mechanism of protrusion formation, we measure several properties of disclination lines as a function of radial position $x_{r}$, with the droplet's center of mass being the reference point of a spherical coordinate system. For approximately ellipsoidal droplet shapes, $x_{r}$ can be used as a proxy to divide the initially spherical droplet of radius $R$ into a bulk domain $\left(x_{r}<R\right)$ and a protrusion domain $\left(x_{r}>R\right)$. Disclination lines in the bulk $\left(x_{r}<R\right)$ of soft droplets undergo chaotic movement $\left(\left\langle\mathbf{v}_{\mathbf{r}}\right\rangle \approx 0\right)$ and are mostly twist type. By contrast, in protrusions $\left(x_{r}>R\right)$, disclination lines mostly consist of $+1 / 2$ line segments (approximately 70\%) and show persistent self-propulsion along the radial protrusion axis $\left[\left\langle\mathbf{v}_{\mathbf{r}}\right\rangle \gg 0\right.$; see Fig. 10(a)]. Disclination lines in protrusions are nearly straight, and their total length $L$ is limited by the width of the protrusion $\left[L\left(x_{r}\right) \approx\right.$ const for $x_{r}>R$; see Fig. 10(b)].

Droplets of sizes much larger than the active length scale $R \gg \ell_{\zeta}$ are strongly deformed, and protrusions do not always grow along the radial axis [Fig. S5(a) [30]], thereby rendering the spherical approximation unsuitable. Still, it can be observed that $+1 / 2$ defect lines are much more frequent in soft droplets with protrusions than in spherical droplets without protrusions [Fig. S5(b) [30]].

The morphology of extensile droplets is determined by two control parameters: The activity number $A=$ $R \sqrt{\zeta / K_{\mathrm{LC}}}$ controls the density of disclination lines inside the bulk of droplets, while the ratio of elastic constant to surface tension $\Psi=K_{\mathrm{LC}} / K_{\varphi}$ quantifies the energetic cost associated with nematic deformations in the bulk compared to deformations of the interface (assuming surface tension dominates the bending stiffness). The morphology of 

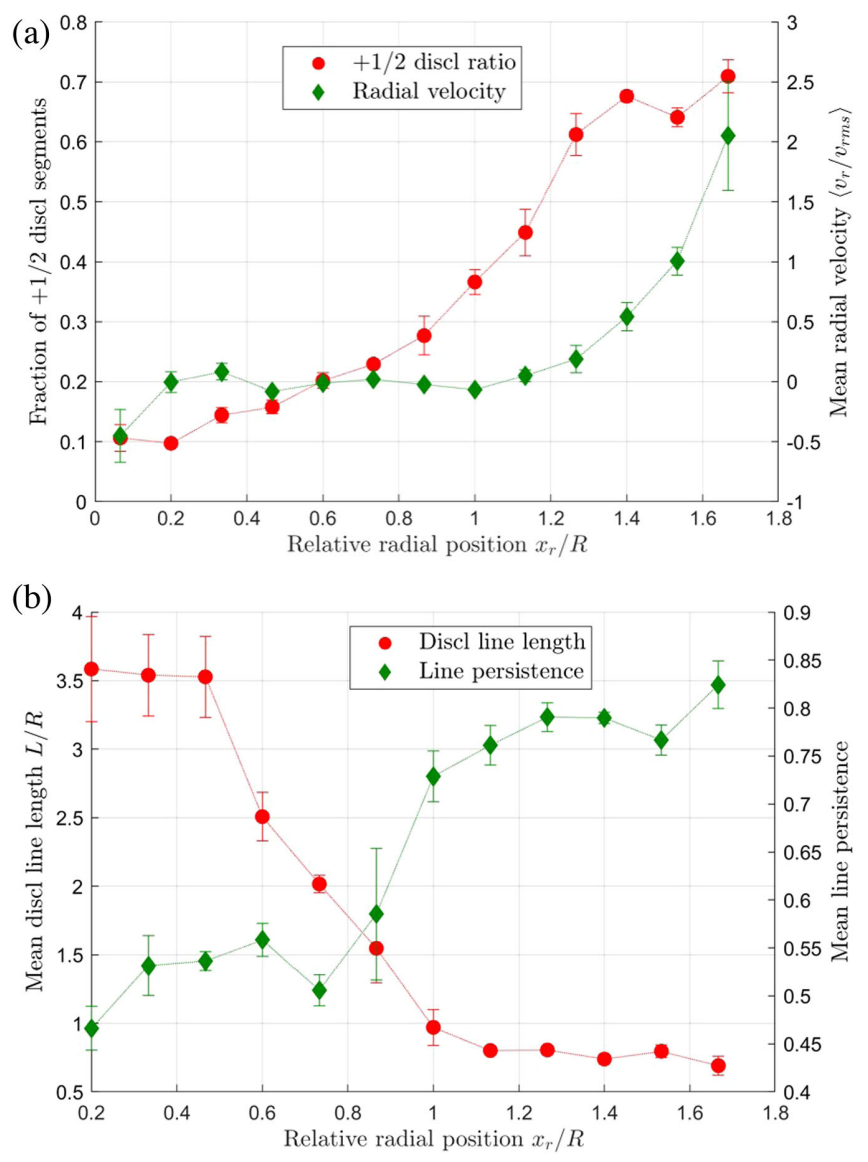

FIG. 10. Disclination line properties for extensile activity as a function of radial position $x_{r}$ in an initially spherical droplet of radius $R$. (a) Most of the disclination lines in protrusions $\left(x_{r}>R\right)$ show $\mathrm{a}+1 / 2$ director configuration for which the self-propulsion velocity $v_{r}$ points radially outward. (b) Disclination lines in protrusions $\left(x_{r}>R\right)$ are nearly straight lines whose length is limited by the diameter of the protrusion. The radial component of the self-propulsion velocity $v_{r}$ is obtained by taking the droplet's center of mass as the reference point of a spherical coordinate system. The fraction of $+1 / 2$ disclination lines is defined as the fraction of disclination line segments with twist angle $3 / 4 \pi \leq \beta \leq \pi$ and the persistence of disclination lines as the ratio of end point distance over total line length. Disclination line properties are calculated by sorting observations $\{Y\}$ into bins of range $X_{i} \pm h / 2$. The position of error bars shows the bin mean $\left\langle\left\{Y_{i}\right\}\right\rangle$, and the size of error bars indicates the standard error of the mean $\epsilon=\operatorname{std}\left(\left\{Y_{i}\right\}\right) / \sqrt{N_{i}}$, where $N_{i}$ is the number of observations in bin $X_{i} \pm h / 2$. The averaging is performed over $T=100000$ time steps, which is much longer than the morphological timescale on which protrusion formation happens (approximately 2000 time steps; see Fig. 9).

droplets as a function of $A$ and $\Psi$ is shown in Fig. 11. For very stiff interfaces $(\Psi \ll 1)$, droplets are nearly spherical and host disclination lines if the activity $A$ is sufficiently large (blue diamonds and purple triangles). Soft interfaces ( $\Psi \geq 1$ ), however, allow the formation of fingerlike protrusions as soon as disclination lines are formed inside

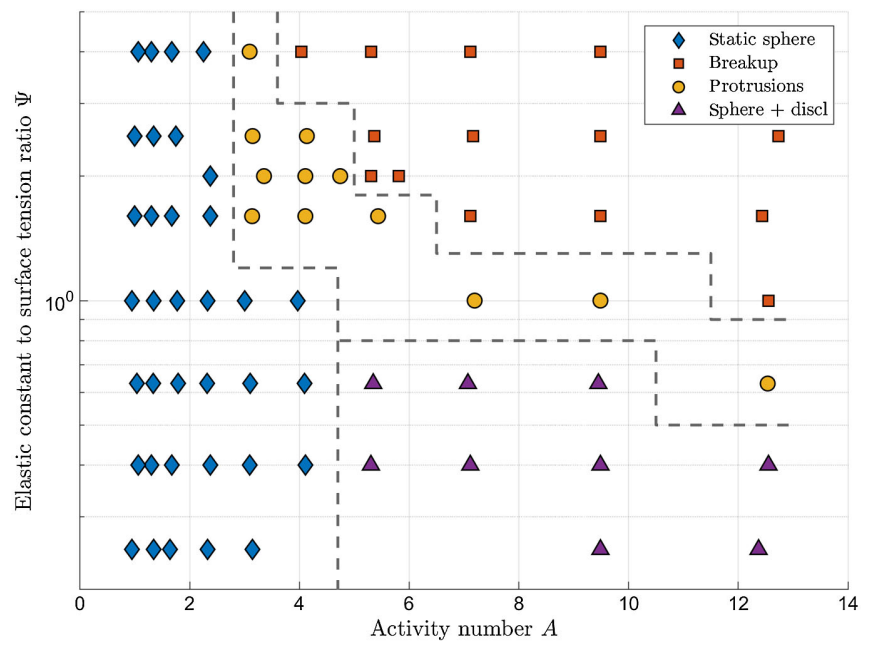

FIG. 11. Morphology diagram of extensile droplets $(\zeta>0)$ as a function of the activity number $A=R \sqrt{\zeta / K_{\mathrm{LC}}}$ and the elastic constant to surface tension ratio $K_{\mathrm{LC}} / K_{\varphi}$. Parameters $\zeta$ and $K_{\mathrm{LC}}$ are varied, while the surface tension and droplet size are fixed to one of the following values: $K_{\varphi} \in\{0.1,0.2\}$ and $R \in\{15,30\}$.

droplets (yellow circles). If active forces become much larger than the passive restoring force of the interface, active flows tear apart the droplet, which breaks up into smaller parts (orange squares; see also Movie S5 [30]). The strength of protrusion formation can be quantified by the gyrification index, which is a function of $A$ and $\Psi$ [Fig. S8(a) [30]].

\section{Contractile activity triggers droplet invagination and surface wrinkles}

We now consider contractile activity which causes droplet invagination or the formation of comb-shaped droplet deformations creating wrinkle patterns on the surface. Unlike protrusion formation in extensile systems, droplet deformations in contractile systems originate from smooth director field deformations and are not mediated via disclination lines.

In contractile systems, active anchoring favors normal surface alignment. Every spherical or topologically equivalent surface with normal surface alignment everywhere enforces the formation of at least one +1 disclination loop in the bulk due to topological constraints. These loops are usually associated with a large elastic energy cost due to strong deformations of the director field and, thus, are observed in drops only if strong normal anchoring is enforced by additional terms in the free energy. Otherwise, in the absence of disclination lines, the director field forms a ring with in-plane surface alignment encircling the droplet [Fig. 12(a)] to maximize the area of perpendicular surface alignment favored by active anchoring while avoiding the formation of $\mathrm{a}+1$ defect loop in the bulk. The ring of in-plane surface alignment is associated with nematic bend deformations in the bulk; thus, we refer 


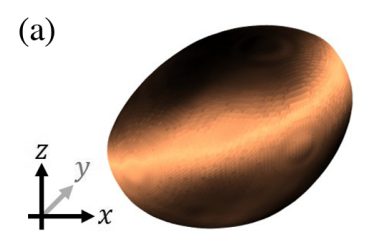

(d)

(b)

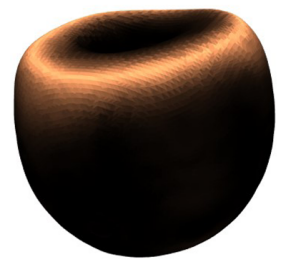

(c)

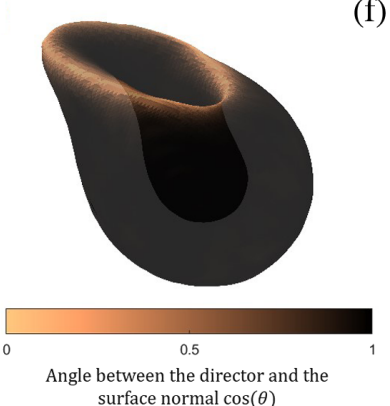

(f)

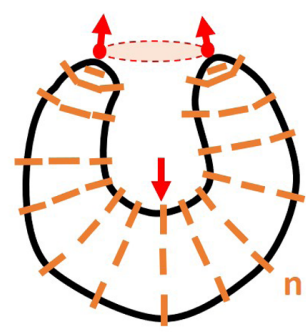

FIG. 12. Initiation of droplet invagination by contractile activity shown by three snapshots at times $t_{1}<t_{2}<t_{3}$. (a)-(c) show the three-dimensional droplet shape with color coding indicating the local surface alignment of the director field. (d)-(f) are schematic diagrams of the director field $\mathbf{n}$, showing the active forces (red arrows) and the position of the bend ring (red, dotted circle). (d) The ring of in-plane alignment at the equator causes nematic bend deformations in the bulk. Contractile activity causes these bend deformations to push outward (red arrows), thus deforming the droplet to an oblate shape. (e) While the ring of in-plane alignment moves toward one of the poles, the bend deformations in the bulk point perpendicular to the surface, which causes the active forces to develop a component pointing toward the pole. This process creates a dent in the center of the ring. (f) Because of the perpendicular surface alignment caused by active anchoring, the dent in the center of the ring leads to a director splay deformation in the bulk. Contractile activity causes this splay deformation to push inward, thus increasing the depth of the dent. Snapshots created from simulation with elastic constant $K_{\mathrm{LC}}=$ 0.4 at times $t_{1}=15000, t_{2}=30000$, and $t_{3}=50000$.

to this structure as a bend ring from now on [Fig. 12(d)]. Contractile activity causes the bend ring to push outward, thereby deforming the droplet to an oblate ellipsoid until passive forces arising from surface tension and membrane rigidity counterbalance the active force.

If active forces are small compared to the passive restoring forces arising from membrane and director field deformations, the oblate bend-ring configuration is stable. Perturbations of ring shape and position are associated with increasing elastic deformations of the membrane and the director field, thereby creating an energy barrier which

stabilizes the configuration (Fig. S6 [30]). The exact free energy profile depends on model parameters such as the surface tension $K_{\varphi}$, membrane rigidity $\kappa$, nematic elastic constant $K_{\mathrm{LC}}$, and bulk properties.

If active forces are sufficiently strong to overcome the passive restoring force, however, the bend ring at the equator is unstable. It contracts while moving toward one of the poles, and its motion results in invagination, leading to a cup-shaped configuration of the droplet.

This instability arises from the active flow set up by the director configuration of the bend ring which pushes outward along the deformation axis [Fig. 1(a)]. Any small deviations from a perfect ellipsoidal droplet shape cause a component of the active flow to point toward one of the poles. The resulting droplet deformation rotates the axis of the bend ring further toward the pole, thereby in turn further increasing the active force component in the direction of the pole [Figs. 12(b) and 12(e)].

As the contracting bend ring approaches the pole, it creates an area of splay deformation in its center due to active anchoring enforcing perpendicular surface alignment [Fig. 12(e)]. This splay deformation sets up an active flow pointing inward [Fig. 1(b)]. The outward-pointing forces of the bend ring together with the inward-pointing forces in its center combine to drive droplet invagination [Figs. 12(c) and 12(f)]. If the magnitude of active stress $\zeta$ is not significantly reduced after complete invagination, this process eventually causes the cavity that is driven into the drop to break off (cup closure), as the passive restoring forces resulting from membrane rigidity and surface tension are not sufficient to compensate the active forces (see Movie S5 [30]). However, if the system is active only for a certain amount of time and $\zeta(t)$ is reduced after the initial invagination, the cup-shaped drop can be stabilized, analogous to the natural course of morphogenetic events, which are controlled by biochemical signals and begin and end at a predefined time [32].

Alternatively, if the interface is too stiff for active forces to initiate drop invagination, droplets perform an active run-and-tumble motion (Fig. 13 and Movie S3 [30]). Instead of a large cavity, only a small dip is formed at the center of the bend ring, where the interface resists further deformations and the splay configuration of the director field leads to flows which push the droplet forward (run phase). Eventually, the director deformations relax via the nucleation of a $\beta=\pi$ half-loop, and the self-propulsive disclination line travels from the position of the dip to the opposing interface. This process reorientates the bend ring along a random direction perpendicular to the axis of the dip (tumble phase), and the process repeats along the new direction. During the run phase, the droplet approximately moves on straight lines, after which it makes a $90^{\circ}$ turn into a random direction. The long time dynamics of the droplet motion is diffusive and resembles a three-dimensional random walk known as a freely rotating chain in polymer 
(a)

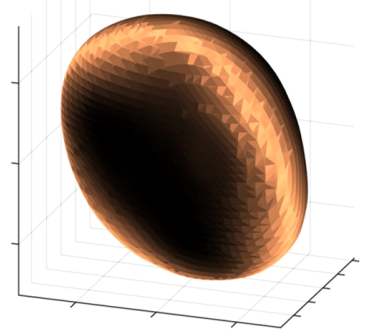

(c)

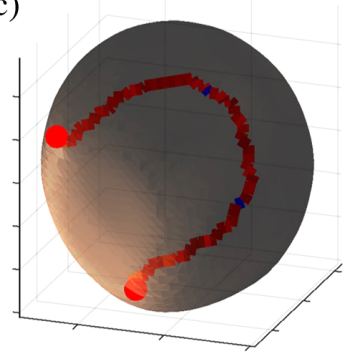

(e)

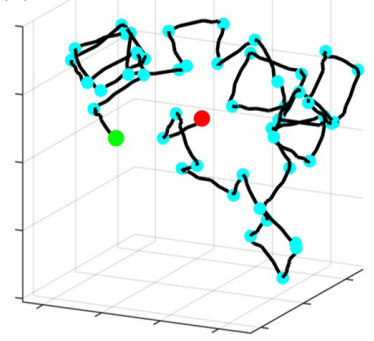

(b)

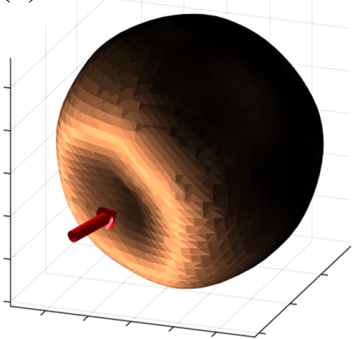

(d)

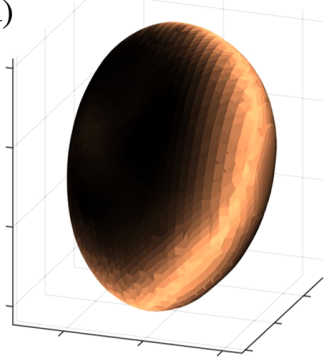

(f)

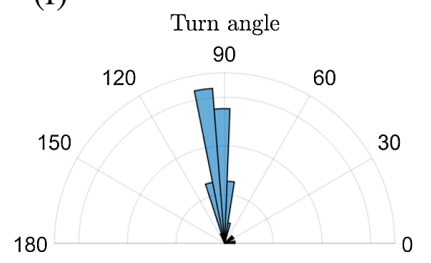

(a)
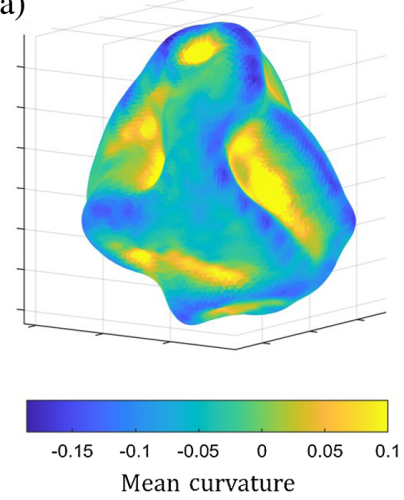

Mean curvature (b)

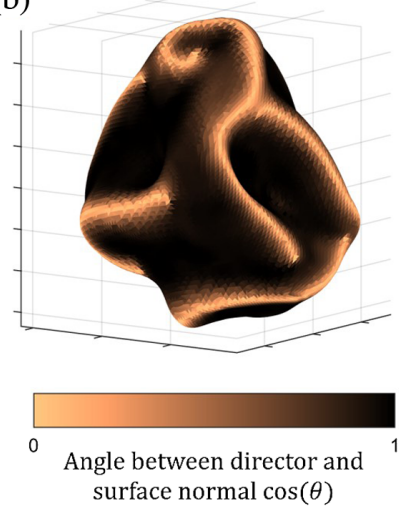

(c)

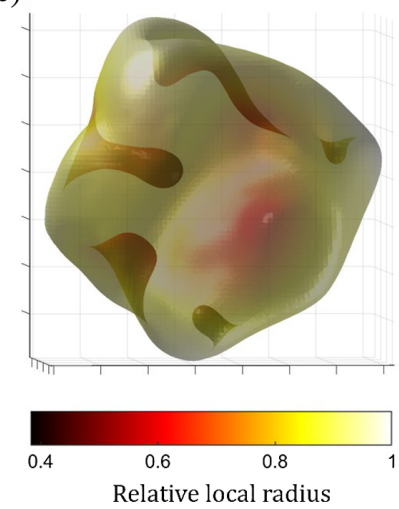

FIG. 14. Snapshots of a contractile drop showing (a) the mean curvature and (b) the orientation of the director field on the surface. Contractile droplets which contain disclination lines show stripes of in-plane director alignment on the surface which connect end points of the disclination lines in the bulk (Fig. 3). Bend and splay deformations of the director field near the surface create comb-shaped deformations along the stripes of in-plane alignment, resulting in a wrinkle pattern. In between stripes of inplane alignment, dimples and valleys are driven into the drop as shown in snapshot (c), which depicts the local radius on a semitransparent drop. The dynamical structure of active surface wrinkles is shown in Movie S4 [30], together with a 3D view of the surface structure. eventually reaches the interface. This event, which we refer to as a tumbling event, reorientates the bend ring along a random direction perpendicular to the axis of the dip, and the process repeats (d). The long time dynamics of the droplet's motion is diffusive and resembles a three-dimensional random walk (e), which is known in polymer physics as a freely rotating chain with a turn angle $\theta=90^{\circ}$ (f). Tumbling events are shown as cyan dots, and the start and end points are shown as red and green dots, respectively. Snapshots and random walk created from simulations with droplet size $R=15$; see also Movie S3 and Fig. S9 [30].

physics with a turn angle $\theta=90^{\circ}$. Tumbling occurs periodically at low activity and becomes more chaotic close to the transition to active turbulence at larger activity (Fig. S9 [30]).

In contractile droplets which are larger than the active length scale $R \gg \ell_{\zeta}$, many disclination lines are present in

the bulk, and the surface shows characteristic stripes of inplane director alignment connecting the end points of disclination lines (Fig. 3). The stripes on the surface are associated with nematic bend deformations in the bulk which induce active forces pushing outward. This process creates comb-shaped deformations along the stripes of in-plane alignment, resulting in a surface wrinkle pattern which we term active wrinkling (Fig. 14 and Movie S4 [30]). In addition, the droplet deformation causes the perpendicularly aligned surface areas between in-plane stripes to form splay deformations in the bulk, which cause inward-pushing, active forces that create dimples and valleys, further enhancing the wrinkle pattern. Along the in-plane ridges the mean curvature is negative, while in the center of holes it is positive. Surface alignment $\cos (\theta)$ is, 


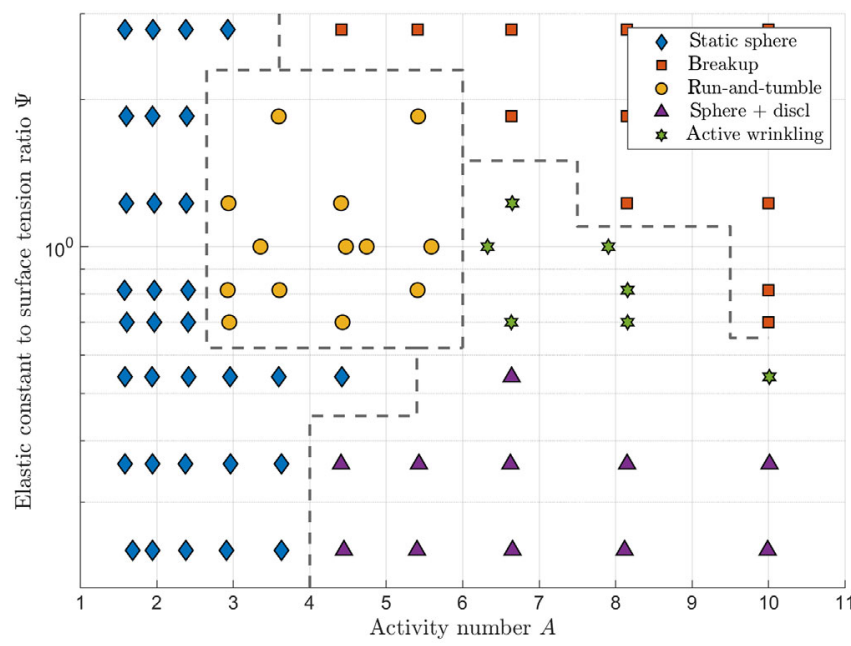

FIG. 15. Morphology diagram of contractile droplets $(\zeta<0)$ as a function of the activity number $A=R \sqrt{\zeta / K_{\mathrm{LC}}}$ and elastic constant to surface tension ratio $K_{\mathrm{LC}} / K_{\varphi}$. There is a smooth transition between spherical droplets with disclination lines and active wrinkling. Parameters $\zeta$ and $K_{\mathrm{LC}}$ are varied, while the surface tension and droplet size are fixed to one of the following values: $K_{\varphi} \in\{0.1,0.2\}$ and $R \in\{15,30\}$.

therefore, correlated to the local mean curvature of the interface with in-plane surface alignment $[\cos (\theta) \approx 0]$ associated with points of negative mean curvature and perpendicular surface alignment $[\cos (\theta) \approx 1]$ predominating at points of positive mean curvature (Fig. S7 [30]).

As for the extensile case, the morphology of contractile droplets is determined by the activity number $A=$ $R \sqrt{\zeta / K_{\mathrm{LC}}}$ and ratio of elastic constant to surface tension $\Psi=K_{\mathrm{LC}} / K_{\varphi}$ (Fig. 15). Very stiff interfaces $(\Psi \ll 1)$ cause droplets to be nearly spherical and host disclination lines if activity $A$ is sufficiently large (blue diamonds and purple triangles). If interfaces are sufficiently soft $(\Psi \approx 1)$, droplets can be either be static, oblate ellipsoids without disclination lines (blue diamonds), perform a run-andtumble motion (yellow circles), or form active surface wrinkles (green stars). For very soft interfaces $(\Psi \gg 1)$, droplets are static ellipsoids at low activity, and full droplet invagination takes place at larger activity, leading the droplet to eventually break up (orange squares). The degree of invagination and wrinkling can also be quantified by a gyrification index, which varies with $A$ and $\Psi$ [Fig. S8(b) [30]].

\section{DISCUSSION}

In this paper, we investigate the morphology and defect dynamics in active deformable droplets in three dimensions, considering both extensile activity, as present in experimental systems such as microtubule-kinesin mixtures [33], human bronchial epithelial cells [34], and MadinDarby canine kidney cells [8], as well as contractile activity, which is found in systems such as mouse fibroblast cells [10] or actomyosin gels [35]. We show that active stresses cause in-plane (extensile) or perpendicular (contractile) surface alignment of the director field at the interface of droplets. Unlike thermodynamic surface anchoring, which involves an anchoring free energy, active anchoring is a hydrodynamic effect due to the flows driven by gradients of the nematic ordering $\mathbf{Q}$ at the interface. Active anchoring has important consequences for the dynamics of disclination lines close to the interface, such as the preferential formation of wedgelike surface disclinations in extensile systems or the creation of in-plane aligned stripes connecting twistlike surface disclinations in contractile systems. This consequence, in turn, triggers the formation of fingerlike protrusions in soft, extensile droplets or the creation of surface wrinkles and droplet invagination in contractile systems. Furthermore, we apply the ideas of 2D active turbulence, such as defect velocities and the existence of an active length scale, to three dimensions. We identify an active length scale $\ell_{\zeta}$ of three-dimensional active turbulence, which controls both the density of disclination lines and the correlation length of the twist angle $\beta$ along disclination lines in the bulk of nematic droplets.

Recent work interprets several 2D biological systems including bacteria biofilms [17,36], epithelial tissue [8], spindle-shaped cell monolayers [10], and actin fibers in regenerating hydra [37] in terms of the theories of active nematics. It is interesting to ask whether similar ideas may prove useful in 3D to describe biological systems in which nematic constituents, such as protein filaments, eukaryotic cells, or bacteria, are organized as solid, 3D structures with a confining interface. There are several examples where cells migrate collectively as a cohesive group, thereby maintaining supracellular properties such as collective force generation and tissue-scale hydrodynamic flow [38-40]. For example, in some modes of collective cell movement implicated in cancer invasion, a blunt budlike tip consisting of multiple cells that variably change position and lack well-defined leader cells protrudes from the tumor $[41,42]$. Hence, the natural appearance of fingerlike protrusions in active nematic droplets could serve as a new approach to explain collective cell invasion of tumors.

In eukaryotic cells, membrane shapes under mechanical stress are mostly controlled by the mechanics of the cortical actin cytoskeleton underlying the cell membrane which produces contractile stresses. It has been observed that membrane shapes mainly depend on the actin thickness, where thin shells show a cup-shaped deformation and thick shells produce membrane wrinkles [43]. Our work provides a possible link between contractile activity and the emergence of cell-surface ruffles, circular dorsal ruffles, and caveolae [44-46] at the surface of cells. Surface invagination which plays a role in active transport through cell membranes might also be related to local contractile forces. During macropinocytosis (fluid endocytosis), extracellular 
fluid is brought into the cell through an invagination of the cell membrane forming a small vesicle inside the cell. Vesicles form from cell-surface ruffles that close first into open cups (ruffle closure) and then into intracellular vesicles (cup closure) [47,48], which is reminiscent of the dimples that are driven into contractile drops. Some morphologies observed in contractile drops, such as partial invagination and run-and-tumble motion, are known to also occur in active polar droplets $[49,50]$. In contrast to nematic systems, which are characterized by a headless director field $\mathbf{n} \leftrightarrow-\mathbf{n}$, polar systems are described by a polarization vector p. As a result, polar models do not exhibit $\pm 1 / 2$ topological defects, which are shown to contribute to the dynamics and flows in cell cultures and bacterial biofilms $[8,10,17,34,36]$.

Up to now, most of the research on three-dimensional active nematics has been focused on the topology and dynamics of closed disclination loops in the bulk or disclination lines in fixed geometries [12-16]. Previous investigations of two-dimensional active nematics which are confined to the surface of a 3D shell explore the connection between 2D topological defects and shell morphology [51]. With this work, we expand the current understanding of three-dimensional active nematics, considering the interplay between motile disclination lines, deformations of the nematic director field, and the droplet interface.

Future directions include the consideration of more complex geometries, in particular, thick, nematic shells or the addition of an anchoring free energy enforcing a given surface alignment. The motivation behind this direction is that most biological systems during embryonic development form sheets or shells rather than solid structures. It will be interesting to return to a more detailed investigation of time-dependent activity $\zeta(t)$ in the future, analogous to the natural course of morphogenetic events, which are controlled by biochemical signals which may vary over time. Another aspect when considering cell aggregates such as embryos or tumors is that they are often composed of different cell types with different mechanical properties and cellular responses [52]. It would be interesting to extend our model to two or more distinct, nematic fluid phases with different mechanical properties and activity coefficients, thereby incorporating cellular heterogeneity within cell aggregates. Another important aspect is that cells are usually surrounded by a threedimensional dense network of macromolecules, the extracellular matrix, which provides structural support and is known to strongly affect cell migration behavior $[53,54]$. The investigation of how a viscoelastic medium or a dense polymer solution affects active droplets therefore appears to be a promising direction.

\section{ACKNOWLEDGMENTS}

We thank Kristian Thijssen for helpful discussions. This project was funded by the European Commissions Horizon
2020 research and innovation program under the Marie Sklodowska-Curie Grant Agreement No. 812780.

\section{APPENDIX A: PHASE-FIELD MODELING OF BIOLOGICAL MEMBRANES}

The elasticity of a two-dimensional cell membrane is usually described by the following curvature energy proposed by Helfrich [20]:

$\mathcal{F}_{H}=\kappa / 2 \int\left(C-C_{0}\right)^{2} d A+\kappa_{G} \int G d A+\gamma \int d A$,

where $C=\left(c_{1}+c_{2}\right)$ and $G=c_{1} c_{2}$ are the total and Gaussian curvatures of the membrane, respectively, given in terms of the principal curvatures $c_{1}$ and $c_{2} . \kappa$ and $\kappa_{G}$ are the bending rigidity and saddle-splay modulus, respectively, $C_{0}$ is the spontaneous curvature caused by any asymmetry in the membrane structure, and $\gamma$ is the surface tension. In our model, we consider a smooth concentration field $\varphi$ associated with a Landau free energy density $f_{\mathrm{GL}}[\varphi]=f_{b}+f_{\mathrm{st}}$ to model the mechanical properties of a droplet. The first part of our Landau free energy includes the membrane bending energy:

$$
f_{b}=\frac{\kappa^{*}}{2}\left(-\varphi+\varphi^{3}-\epsilon^{2} \nabla^{2} \varphi\right)^{2} .
$$

This free energy can be minimized analytically along the surface normal direction $z$ in equilibrium, resulting in a smooth concentration profile $\varphi_{0}(z)=\tanh (z / \ell)$ connecting phases $\varphi=-1$ and $\varphi=+1$ over a characteristic width $\ell=\sqrt{2} \epsilon$. Given the profile $\varphi_{0}(z)$, we can compare the free energies of the phase-field representation and classical Helfrich theory to obtaining a mapping between model parameters [19]:

$$
\gamma=0, \quad C_{0}=0, \quad \kappa_{G}=0, \quad \kappa=\frac{2 \epsilon^{3}}{3 \sqrt{2}} \kappa^{*},
$$

which includes only a bending contribution $(\kappa>0)$ of the membrane. To include surface tension, we need to add the following term to the free energy:

$$
f_{\mathrm{st}}=\frac{K_{\varphi}}{2}(\nabla \varphi)^{2} .
$$

It should be noted that the shape of $\varphi_{0}(z)$ coincides with the equilibrium profile of the well-known Cahn-Hilliard equation, which is usually used to describe phase separation in systems with surface tension and without bending rigidity. If the interface is narrow, then $\left(\nabla \varphi_{0}\right)^{2}$ behaves as a $\delta$ function, $\lim _{\ell \rightarrow 0}(3 / 4 \ell) \operatorname{sech}^{4}(z / \ell)=\delta(z)$, and the volume integral over $f_{\text {st }}$ converges to a surface integral over the interface: 


$$
\gamma \int d \mathbf{S}=\gamma \int \delta\left(z-z^{\prime}\right) d \mathbf{V} \approx \frac{3 \ell}{4} \gamma \int\left(\nabla \varphi_{0}\right)^{2} d \mathbf{V}
$$

where $z^{\prime}$ is the position of the interface. Thus, the membrane surface tension is related to the phase-field parameters via $\gamma=\left(2 K_{\varphi} / 3 \ell\right)$, where $\ell=\sqrt{2} \epsilon$ is the width of the membrane. However, it should be noted that this equation is valid only as long as the bending term $f_{b}$ is dominant and $\epsilon$ sets the length scale of the membrane width. If the term for the surface tension $f_{\text {st }}$ is dominant $\left(K_{\varphi} / \kappa^{*}>\epsilon^{2}\right)$, the characteristic width of the equilibrium concentration profile $\varphi_{0}(z)$ is given by $\ell \sim \sqrt{K_{\varphi} / \kappa^{*}}$, and, thus, the surface tension scales as $\gamma \sim \sqrt{K_{\varphi}}$.

Using phase-field theories to model droplets and membranes does not necessarily need that the interface width $\ell$ is on the same scale as the width of the physical interface, but it is sufficient to require that $\ell$ is much smaller than any other length of the system, such as the active length scale $\ell_{\zeta}$ or droplet size $R$.

\section{APPENDIX B: MAPPING OF LB UNITS TO FULL UNITS}

In order to map LB simulation parameters to dimensional quantities in physical units, one requires a physical reference scale for three independent LB parameters, such as lattice spacing $\Delta x$, time step $\Delta t$, and viscosity. For modeling the organization of three-dimensional, multicellular spheroids, the lattice spacing corresponds to the size of individual cells $\Delta x \approx 10 \mu \mathrm{m}$. Assuming disclinations in $3 \mathrm{D}$ move with similar velocities to $+1 / 2$ defects in $2 \mathrm{D}$ cell monolayers, we can compare the velocity of motile $+1 / 2$ disclination lines in our simulation $v_{\mathrm{SP}}^{\mathrm{sim}}=0.002 \Delta x / \Delta t$ with typical flow velocities around $+1 / 2$ defects in experiments $v_{\mathrm{SP}}^{\exp } \approx 20 \mu \mathrm{m} / \mathrm{h}$ [2], which yields $\Delta t=4 \mathrm{~s}$. We run most simulations for up to 250000 time steps, which corresponds to an observation window of $T \approx 12 d$. If we choose the shear viscosity of embryonic tissues $\eta \approx 14 \mathrm{kPa}$ s [55,56] as a viscous reference scale, the LB units are mapped to the following physical units:

\begin{tabular}{lcc}
\hline Parameters & LB units & Full units \\
\hline Lattice spacing $\Delta x$ & 1 & $10 \mu \mathrm{m}$ \\
Time step $\Delta t$ & 1 & $4 \mathrm{~s}$ \\
Viscosity $\eta$ & $1 / 3$ & $14 \mathrm{kPa} \mathrm{s}$ \\
Droplet radius $R$ & $15-30$ & $150-300 \mu \mathrm{m}$ \\
Interface width $\epsilon$ & 1.4 & $14 \mu \mathrm{m}$ \\
Surface tension $\gamma \approx(2 / 3 \epsilon) K_{\varphi}$ & $0.05-0.19$ & $0.5-2 \times 10^{-2} \mathrm{~N} / \mathrm{m}$ \\
Bending rigidity $\kappa \approx\left(\sqrt{2} \epsilon^{3} / 3\right) \kappa^{*}$ & 0.13 & $1.4 \times 10^{-13} \mathrm{Nm}$ \\
Elastic const $K_{\mathrm{LC}}$ & $0.1-0.2$ & $1-2 \times 10^{-7} \mathrm{~N}$ \\
Active stress $\zeta$ & $0.01-0.02$ & $100-200 \mathrm{~Pa}$ \\
Rotational diffusivity $\Gamma_{Q}$ & 0.1 & $2 \times 10^{-6}(\mathrm{Pas})^{-1}$ \\
Mobility $\Gamma_{\varphi}$ & 0.2 & $2 \times 10^{-15} \mathrm{~m}^{2} / \mathrm{Pas}$ \\
Nematic bulk energy $A_{\mathrm{LC}}$ & $0.75-1.5$ & $7.5-15 \mathrm{kPa}$ \\
Droplet bulk energy $A_{\varphi}$ & 0.1 & $1 \mathrm{kPa}$ \\
\hline
\end{tabular}

These parameters are in good agreement with mechanical properties of cell aggregates measured in experiments. Assuming the width of the interface giving rise to surface tension is about the size of individual cells, $\epsilon=14 \mu \mathrm{m}$, the resulting surface tension $\gamma \sim 10^{-2} \mathrm{~N} / \mathrm{m}$ [55,57-59] and bending rigidity $\kappa \sim 10^{-13} \mathrm{~N} \mathrm{~m}[60,61]$ are within the range of values found for tissues and epithelial monolayers. Living cells embedded in a 3D matrix typically generate long-ranged contractile stress of the order of $\zeta \approx 200 \mathrm{~Pa}$ close to the cell [62].

To estimate $K_{\mathrm{LC}}$ in experiments, we need to relate this effective constant of the nematic description to mechanical properties of individual cells. If we assume that cells in a dense aggregate must physically deform when the nematic order is distorted, the elastic energy associated with cell shape deformations is related to the cells' Young modulus $E$ and cell size $\Delta x$. From dimensional arguments, it follows that $K_{\mathrm{LC}} \sim E \cdot \Delta x^{2}$ [2]. The typical Young's modulus of fibroblasts, astrocytes, and some endothelial cells is of the order of $E \sim \mathrm{kPa}[63,64]$, which yields $K_{\mathrm{LC}} \sim 10^{-7} \mathrm{~N}$.

It should be noted that mechanical properties of cell aggregates may vary greatly as the cells' Young modulus varies over several orders of magnitude, $E \sim 0.1-100 \mathrm{kPa}$ for different cell types [64]. Similar variations can be observed for the effective viscosity of tissues, as some viscoelastic cell aggregates are shown to have an effective viscosity up to $\eta \sim 100 \mathrm{kPas}[57,58,65]$.

\section{APPENDIX C: DETECTION OF DISCLINATION LINES AND CALCULATION OF TWIST ANGLE $\beta$}

To find defect positions on a three-dimensional grid, we use Zapotoky's defect-finding algorithm for two-dimensional systems [66], which checks if a disclination is located at the intersection of four voxels forming a $2 \times 2$ square, extended to three-dimensional grids. This extended algorithm checks if a disclination is located at a given voxel by repeating the two-dimensional approach along all three coordinate axes [67]. When a disclination is found, its rotation vector $\Omega$ is determined by taking the cross product of each pair of directors around it. Once all grid points are classified, defect positions are sorted to form continuous lines. A shortcoming of this algorithm is that $\Omega$ has an arbitrary sign; hence, it cannot directly distinguish between $\beta=0(-1 / 2$-type $)$ and $\beta=\pi(+1 / 2$-type) disclinations. To achieve this sorting, we calculate the saddle-splay energy

$$
\bar{f}_{24}=\nabla \cdot[(\mathbf{n} \cdot \nabla) \mathbf{n}-\mathbf{n}(\nabla \cdot \mathbf{n})],
$$

which is negative at a $\beta=\pi$ disclination line segment, positive at a $\beta=0$ segment, and zero for a local twist profile [68]. A similar approach based on a purely local calculation of $\Omega$ is used in related work which finds that this algorithm agrees well with nonlocal methods which 
compute $\Omega$ from the director $\mathbf{n}$ in a small circuit around the disclination of interest (see Supplemental Material of Ref. [12]).

The color jumps seen for some disclination line segments in Figs. 6(a) and 13(c) occur because the calculation of the local tangent of disclination lines is performed on a discrete grid; hence, the local tangent does not vary continuously along a disclination line, especially at points of large curvature. This discontinuity can sometimes create sudden color jumps, as the twist angle $\beta$ depends on both the rotation vector $\Omega$ and the local tangent. Another error source is that the algorithm used to distinguish between $+1 / 2$ and $-1 / 2$-type disclination lines is not perfect and sometimes misclassifies line segments [see two blue line segments in Fig. 13(c) which are falsely classified as $-1 / 2]$. However, neither of the error sources meaningfully impacts the statistics, as the discretization error causes only small jumps $\Delta \cos (\beta)<0.2$ and $\pm 1 / 2$ misclassifications of line segments are very rare $(<5 \%)$.

[1] V. Narayan, S. Ramaswamy, and N. Menon, Long-Lived Giant Number Fluctuations in a Swarming Granular Nematic, Science 317, 105 (2007).

[2] G. Duclos, S. Garcia, H. Yevick, and P. Silberzan, Perfect Nematic Order in Confined Monolayers of Spindle-Shaped Cells, Soft Matter 10, 2346 (2014).

[3] A. Doostmohammadi, J. Ignés-Mullol, J. M. Yeomans, and F. Sagués, Active Nematics, Nat. Commun. 9, 3246 (2018).

[4] M. C. Marchetti, J.-F. Joanny, S. Ramaswamy, T. B. Liverpool, J. Prost, M. Rao, and R. A. Simha, Hydrodynamics of Soft Active Matter, Rev. Mod. Phys. 85, 1143 (2013).

[5] R. Zhang, Y. Zhou, M. Rahimi, and J. J. De Pablo, Dynamic Structure of Active Nematic Shells, Nat. Commun. 7, 13483 (2016).

[6] F. C. Keber, E. Loiseau, T. Sanchez, S. J. DeCamp, L. Giomi, M. J. Bowick, M. C. Marchetti, Z. Dogic, and A. R. Bausch, Topology and Dynamics of Active Nematic Vesicles, Science 345, 1135 (2014).

[7] G. Lee, M. J. Rust, M. Das, R. J. McGorty, J. L. Ross, and R. M. Robertson-Anderson, Myosin-Driven ActinMicrotubule Networks Exhibit Self-Organized Contractile Dynamics, bioRxiv (to be published).

[8] T. B. Saw, A. Doostmohammadi, V. Nier, L. Kocgozlu, S. Thampi, Y. Toyama, P. Marcq, C. T. Lim, J. M. Yeomans, and B. Ladoux, Topological Defects in Epithelia Govern Cell Death and Extrusion, Nature (London) 544, 212 (2017).

[9] R. Mueller, J. M. Yeomans, and A. Doostmohammadi, Emergence of Active Nematic Behavior in Monolayers of Isotropic Cells, Phys. Rev. Lett. 122, 048004 (2019).

[10] G. Duclos, C. Erlenkämper, J.-F. Joanny, and P. Silberzan, Topological Defects in Confined Populations of SpindleShaped Cells, Nat. Phys. 13, 58 (2017).

[11] T. B. Saw, W. Xi, B. Ladoux, and C. T. Lim, Biological Tissues as Active Nematic Liquid Crystals, Adv. Mater. 30, 1802579 (2018).
[12] G. Duclos, R. Adkins, D. Banerjee, M. S. Peterson, M. Varghese, I. Kolvin, A. Baskaran, R. A. Pelcovits, T. R. Powers, A. Baskaran et al., Topological Structure and Dynamics of Three-Dimensional Active Nematics, Science 367, 1120 (2020).

[13] J. Binysh, Ž. Kos, S. Čopar, M. Ravnik, and G. P. Alexander, Three-Dimensional Active Defect Loops, Phys. Rev. Lett. 124, 088001 (2020).

[14] S. Čopar, J. Aplinc, Ž. Kos, S. Žumer, and M. Ravnik, Topology of Three-Dimensional Active Nematic Turbulence Confined to Droplets, Phys. Rev. X 9, 031051 (2019).

[15] L. N. Carenza, G. Gonnella, D. Marenduzzo, and G. Negro, Rotation and Propulsion in 3D Active Chiral Droplets, Proc. Natl. Acad. Sci. U.S.A. 116, 22065 (2019).

[16] L. Carenza, G. Gonnella, D. Marenduzzo, and G. Negro, Chaotic and Periodical Dynamics of Active Chiral Droplets, Physica A (to be published).

[17] Y. I. Yaman, E. Demir, R. Vetter, and A. Kocabas, Emergence of Active Nematics in Chaining Bacterial Biofilms, Nat. Commun. 10, 2285 (2019).

[18] C. Blanch-Mercader and J. Casademunt, Hydrodynamic Instabilities, Waves and Turbulence in Spreading Epithelia, Soft Matter 13, 6913 (2017).

[19] G. R. Lázaro, I. Pagonabarraga, and A. HernándezMachado, Phase-Field Theories for Mathematical Modeling of Biological Membranes, Chem. Phys. Lipids 185, 46 (2015).

[20] W. Helfrich, Elastic Properties of Lipid Bilayers: Theory and Possible Experiments, Z. Naturforsch. C 28, 693 (1973).

[21] P.-G. De Gennes and J. Prost, The Physics of Liquid Crystals (Oxford University, New York, 1993), Vol. 83.

[22] A. Doostmohammadi, S. P. Thampi, and J. M. Yeomans, Defect-Mediated Morphologies in Growing Cell Colonies, Phys. Rev. Lett. 117, 048102 (2016).

[23] M. L. Blow, S. P. Thampi, and J. M. Yeomans, Biphasic, Lyotropic, Active Nematics, Phys. Rev. Lett. 113, 248303 (2014).

[24] J. W. Cahn and J. E. Hilliard, Free Energy of a Nonuniform System. I. Interfacial Free Energy, J. Chem. Phys. 28, 258 (1958).

[25] A. N. Beris and B. J. Edwards, Thermodynamics of Flowing Systems with Internal Microstructure, Oxford engineering science series Vol. 36 (Oxford University, New York, 1994).

[26] D. Marenduzzo, E. Orlandini, and J. M. Yeomans, Hydrodynamics and Rheology of Active Liquid Crystals: A Numerical Investigation, Phys. Rev. Lett. 98, 118102 (2007).

[27] R. A. Simha and S. Ramaswamy, Hydrodynamic Fluctuations and Instabilities in Ordered Suspensions of Self-Propelled Particles, Phys. Rev. Lett. 89, 058101 (2002).

[28] D. Marenduzzo, E. Orlandini, M. E. Cates, and J. M. Yeomans, Steady-State Hydrodynamic Instabilities of Active Liquid Crystals: Hybrid Lattice Boltzmann Simulations, Phys. Rev. E 76, 031921 (2007).

[29] S. Thampi and J. Yeomans, Active Turbulence in Active Nematics, Eur. Phys. J. Special Topics 225, 651 (2016). 
[30] See Supplemental Material at http://link.aps.org/ supplemental/10.1103/PhysRevX.11.021001 for movie captions and Supplemental figures.

[31] T. N. Shendruk, K. Thijssen, J. M. Yeomans, and A. Doostmohammadi, Twist-Induced Crossover from TwoDimensional to Three-Dimensional Turbulence in Active Nematics, Phys. Rev. E 98, 010601(R) (2018).

[32] J. Rozman, M. Krajnc, and P. Ziherl, Collective Cell Mechanics of Epithelial Shells with Organoid-like Morphologies, Nat. Commun. 11, 3805 (2020).

[33] T. Sanchez, D. T. Chen, S. J. DeCamp, M. Heymann, and Z. Dogic, Spontaneous Motion in Hierarchically Assembled Active Matter, Nature (London) 491, 431 (2012).

[34] C. Blanch-Mercader, V. Yashunsky, S. Garcia, G. Duclos, L. Giomi, and P. Silberzan, Turbulent Dynamics of Epithelial Cell Cultures, Phys. Rev. Lett. 120, 208101 (2018).

[35] M. Schuppler, F. C. Keber, M. Kröger, and A. R. Bausch, Boundaries Steer the Contraction of Active Gels, Nat. Commun. 7, 13120 (2016).

[36] D. DellArciprete, M. Blow, A. Brown, F. Farrell, J. S. Lintuvuori, A. McVey, D. Marenduzzo, and W. C. Poon, A Growing Bacterial Colony in Two Dimensions as an Active Nematic, Nat. Commun. 9, 1 (2018).

[37] Y. Maroudas-Sacks, L. Garion, L. Shani-Zerbib, A. Livshits, E. Braun, and K. Keren, Topological Defects in the Nematic Order of Actin Fibers as Organization Centers of Hydra Morphogenesis, bioRxiv (to be published).

[38] A. Shellard and R. Mayor, Supracellular MigrationBeyond Collective Cell Migration, J. Cell Sci. 132, 1 (2019).

[39] P. Friedl and D. Gilmour, Collective Cell Migration in Morphogenesis, Regeneration and Cancer, Nat. Rev. Mol. Cell Biol. 10, 445 (2009).

[40] B. He, K. Doubrovinski, O. Polyakov, and E. Wieschaus, Apical Constriction Drives Tissue-Scale Hydrodynamic Flow to Mediate Cell Elongation, Nature (London) 508, 392 (2014).

[41] P. Friedl and S. Alexander, Cancer Invasion and the Microenvironment: Plasticity and Reciprocity, Cell 147, 992 (2011).

[42] A. J. Ewald, A. Brenot, M. Duong, B. S. Chan, and Z. Werb, Collective Epithelial Migration and Cell Rearrangements Drive Mammary Branching Morphogenesis, Dev. Cell 14, 570 (2008).

[43] R. Kusters, C. Simon, R. L. Dos Santos, V. Caorsi, S. Wu, J.-F. Joanny, P. Sens, and C. Sykes, Actin Shells Control Buckling and Wrinkling of Biomembranes, Soft Matter 15, 9647 (2019).

[44] R. Buccione, J. D. Orth, and M. A. McNiven, Foot and Mouth: Podosomes, Invadopodia and Circular Dorsal Ruffles, Nat. Rev. Mol. Cell Biol. 5, 647 (2004).

[45] J.-L. Hoon, W.-K. Wong, and C.-G. Koh, Functions and Regulation of Circular Dorsal Ruffles, Mol. Cell. Biol. 32, 4246 (2012).

[46] A. Echarri and M. A. Del Pozo, Caveolae-Mechanosensitive Membrane Invaginations Linked to Actin Filaments, J. Cell Sci. 128, 2747 (2015).

[47] J. A. Swanson, Shaping Cups into Phagosomes and Macropinosomes, Nat. Rev. Mol. Cell Biol. 9, 639 (2008).
[48] G. J. Doherty and H. T. McMahon, Mechanisms of Endocytosis, Annu. Rev. Biochem. 78, 857 (2009).

[49] E. Tjhung, M. E. Cates, and D. Marenduzzo, Contractile and Chiral Activities Codetermine the Helicity of Swimming Droplet Trajectories, Proc. Natl. Acad. Sci. U.S.A. 114, 4631 (2017).

[50] E. Tjhung, D. Marenduzzo, and M. E. Cates, Spontaneous Symmetry Breaking in Active Droplets Provides a Generic Route to Motility, Proc. Natl. Acad. Sci. U.S.A. 109, 12381 (2012).

[51] L. Metselaar, J. M. Yeomans, and A. Doostmohammadi, Topology and Morphology of Self-Deforming Active Shells, Phys. Rev. Lett. 123, 208001 (2019).

[52] A. Marusyk and K. Polyak, Tumor Heterogeneity: Causes and Consequences, Biochim. Biophys. Acta 1805, 105 (2010).

[53] M. Ehrbar, A. Sala, P. Lienemann, A. Ranga, K. Mosiewicz, A. Bittermann, S. Rizzi, F. E. Weber, and M. Lutolf, Elucidating the Role of Matrix Stiffness in $3 D$ Cell Migration and Remodeling, Biophys. J. 100, 284 (2011).

[54] A. Pathak and S. Kumar, Independent Regulation of Tumor Cell Migration by Matrix Stiffness and Confinement, Proc. Natl. Acad. Sci. U.S.A. 109, 10334 (2012).

[55] G. Forgacs, R. A. Foty, Y. Shafrir, and M. S. Steinberg, Viscoelastic Properties of Living Embryonic Tissues: A Quantitative Study, Biophys. J. 74, 2227 (1998).

[56] C. Duclut, N. Sarkar, J. Prost, and F. Jülicher, Fluid Pumping and Active Flexoelectricity Can Promote Lumen Nucleation in Cell Assemblies, Proc. Natl. Acad. Sci. U.S.A. 116, 19264 (2019).

[57] T. V. Stirbat, A. Mgharbel, S. Bodennec, K. Ferri, H. C. Mertani, J.-P. Rieu, and H. Delanoë-Ayari, Fine Tuning of Tissues' Viscosity and Surface Tension through Contractility Suggests a New Role for $\alpha$-Catenin, PLoS One 8, e52554 (2013).

[58] K. Guevorkian, M.-J. Colbert, M. Durth, S. Dufour, and F. Brochard-Wyart, Aspiration of Biological Viscoelastic Drops, Phys. Rev. Lett. 104, 218101 (2010).

[59] A. Mgharbel, H. Delanoë-Ayari, and J.-P. Rieu, Measuring Accurately Liquid and Tissue Surface Tension with a Compression Plate Tensiometer, HFSP J. 3, 213 (2009).

[60] J. Fouchard, T. P. Wyatt, A. Proag, A. Lisica, N. Khalilgharibi, P. Recho, M. Suzanne, A. Kabla, and G. Charras, Curling of Epithelial Monolayers Reveals Coupling between Active Bending and Tissue Tension, Proc. Natl. Acad. Sci. U.S.A. 117, 9377 (2020).

[61] A. Trushko, I. Di Meglio, A. Merzouki, C. BlanchMercader, S. Abuhattum, J. Guck, K. Alessandri, P. Nassoy, K. Kruse, B. Chopard et al., Buckling of Epithelium Growing under Spherical Confinement, bioRxiv:513119.

[62] Y. L. Han, P. Ronceray, G. Xu, A. Malandrino, R. D. Kamm, M. Lenz, C. P. Broedersz, and M. Guo, Cell Contraction Induces Long-Ranged Stress Stiffening in the Extracellular Matrix, Proc. Natl. Acad. Sci. U.S.A. 115, 4075 (2018).

[63] J. Solon, I. Levental, K. Sengupta, P. C. Georges, and P. A. Janmey, Fibroblast Adaptation and Stiffness Matching to Soft Elastic Substrates, Biophys. J. 93, 4453 (2007). 
[64] T. G. Kuznetsova, M. N. Starodubtseva, N. I. Yegorenkov, S. A. Chizhik, and R. I. Zhdanov, Atomic Force Microscopy Probing of Cell Elasticity, Micron 38, 824 (2007).

[65] P. Marmottant, A. Mgharbel, J. Käfer, B. Audren, J.-P. Rieu, J.-C. Vial, B. Van Der Sanden, A. F. Marée, F. Graner, and H. Delanoë-Ayari, The Role of Fluctuations and Stress on the Effective Viscosity of Cell Aggregates, Proc. Natl. Acad. Sci. U.S.A. 106, 17271 (2009).
[66] M. Zapotocky, P. M. Goldbart, and N. Goldenfeld, Kinetics of Phase Ordering in Uniaxial and Biaxial Nematic Films, Phys. Rev. E 51, 1216 (1995).

[67] J. Hobdell and A. Windle, A Numerical Technique for Predicting Microstructure in Liquid Crystalline Polymers, Liq. Cryst. 23, 157 (1997).

[68] L. Tran, M. O. Lavrentovich, D. A. Beller, N. Li, K. J. Stebe, and R.D. Kamien, Lassoing Saddle Splay and the Geometrical Control of Topological Defects, Proc. Natl. Acad. Sci. U.S.A. 113, 7106 (2016). 